\title{
Platelets: A Multiscale Approach for Recovering Edges and Surfaces in Photon Limited Medical Imaging
}

\author{
Rebecca M. Willett, Student Member, IEEE, and Robert D. Nowak, Member, IEEE
}

\begin{abstract}
This paper proposes a new multiscale image decomposition based on platelets. Platelets are localized functions at various scales, locations, and orientations that produce piecewise linear image approximations. Platelets are well suited for approximating images consisting of smooth regions separated by smooth boundaries. For smoothness measured in certain Hölder classes, it is shown that the error of m-term platelet approximations can decay significantly faster than that of $\mathrm{m}$-term approximations in terms of sinusoids or wavelets. This suggests that platelets may outperform existing techniques for image denoising and reconstruction. Moreover, the platelet decomposition is based on a recursive image partitioning scheme which, unlike conventional wavelet decompositions, is very well suited to photon-limited medical imaging applications involving Poisson distributed data. Fast, platelet-based, maximum penalized likelihood methods for photon-limited image denoising, deblurring and tomographic reconstruction problems are developed. Because platelet decompositions of Poisson distributed images are tractable and computationally efficient, existing image reconstruction methods based on expectation-maximization type algorithms can be easily enhanced with platelet techniques. Experimental results demonstrate that platelet-based methods can outperform standard reconstruction methods currently in use in confocal microscopy image restoration and emission tomography.
\end{abstract}

Keywords: wavelets, multiresolution, denoising, tomography

${ }^{*}$ Corresponding author: R. Nowak. The authors are with the Department of Electrical and Computer Engineering, 2041 Duncan Hall, Rice University, Houston, TX, USA (e-mail: \{willett, nowak\}@ece.rice.edu, phone: 713 3486318 , fax: 7133486196$)$.

R. Nowak was partially supported by the National Science Foundation, grant no. MIP-9701692, the Army Research Office, grant no. DAAD19-99-1-0349, the Office of Naval Research, grant no. N00014-00-1-0390. R. Willett was partially supported by the National Science Foundation Graduate Student Fellowship. 


\section{Photon-Limited Medical Imaging}

Many medical imaging modalities involve the detection of (light or higher energy) photons, and often the random nature of photon emission and detection is the dominant source of noise in imaging systems. Such cases are referred to as photon-limited imaging applications, since the relatively small number of detected photons is the factor limiting the signal-to-noise ratio. These applications include Positron Emission Tomography (PET), Single Photon Emission Computed Tomography (SPECT), Confocal Microscopy, and Infrared (IR) imaging [1], [2], [3]. The data collected by these imaging systems are usually assumed to obey a spatial Poisson distribution involving a two-dimensional intensity image that describes the probability of photon emissions at different locations in space. The mean and variance of a Poisson process are equal to the intensity. The intensity/mean is the "signal" of interest and the variability of the data about the mean can be interpreted as "noise." Thus, as the intensity varies spatially as a function of anatomy, structure, or function, so does the signal-to-noise ratio. In this sense it could be said that the noise in photon-limited imaging is signal-dependent.

Statistical methods for photon-limited image deblurring and reconstruction are especially effective since they can account for the special properties of the Poisson distribution. The maximum likelihood estimator (MLE) is the most popular statistical tool and is routinely applied in scientific and clinical practice. In most cases the MLE must be computed numerically, and the most common method for this purpose is the expectation-maximization (EM) algorithm [4], [5], [6] (also known as the Richardson-Lucy algorithm in the context of Poisson data [7]). EM algorithms have been widely studied and applied and provide a very simple means to compute the MLE. However the maximum likelihood criterion is not always useful. For example, in PET and SPECT the resulting system of equations is very ill-posed and often the MLE is extremely noisy (highly variable). The EM algorithm may even diverge.

To combat this problem the maximum likelihood criterion can be replaced with maximum penalized likelihood criteria [8], [9], [10], [11]. These criteria are constructed by adding a penalizing function to the Poisson log-likelihood function. The penalizing function measures the smooth- 
ness of the intensity image and assigns weaker penalties to smoother intensities and stronger penalties to more irregular intensities. Assumptions of smoothness are not unreasonable in practice. For example, the uptakes of radioactive pharmaceuticals are fairly uniform in regions of homogeneous tissue, resulting in smoothly varying intensities within organs. Basic anatomical and physiological considerations suggest that extremely irregular intensities are unnatural.

The penalizing function can be specified by an ad hoc smoothness measure, a Bayesian prior distribution for the intensity image [8], [9], or a complexity measure [12], [13]. Smoothness measures include simple quadratic functions that measure the similarity between the intensity values of neighboring pixels, as well as non-quadratic measures that better preserve edges. Similar penalty functions result from Markov Random Field (MRF) priors. Complexity measures are usually associated with an expansion of the intensity image with respect to a set of basis functions (e.g., Fourier or wavelet) and count the number of terms retained in a truncated or pruned series [14], [15]; the more terms (basis functions) used to represent the image, the higher the complexity measure. An intensity that maximizes a penalized likelihood criterion is called a maximum penalized likelihood estimator (MPLE). Many algorithms (often EM algorithms or close relatives) have been developed to compute MPLEs under various penalization schemes [16].

An alternative to MPLE-based methods is the "stopped" EM-MLE solution [17]. The idea here is to stop the iterations of the EM-MLE algorithm at a suitable point, before it converges to the undesirable MLE or diverges. The stopped EM-MLE algorithm implicitly produces a smooth solution and is perhaps the most widely applied approach in practice. The popularity of this procedure is probably due to its simplicity and the availability of very fast EM-type algorithms for the basic maximum likelihood criterion. This paper hopes to convince the reader that MPLEs based on complexity penalized multiscale (or "multiresolution") image representations not only compare favorably with EM-MLE reconstructions in terms of computational speed, but also can provide reconstructions that are significantly superior to the best stopped EM-MLE solution.

Many investigators have considered the use of wavelet representations for image denoising, deblurring, and tomographic image reconstruction; for examples, see [18], [19], [20], [21], [22], 
[23], [24]. However, in the context of photon-limited imaging most wavelet-based approaches are based on Gaussian or other simplifying approximations to the Poisson likelihood. This is due to the fact that it is very difficult in general to apply wavelets (as well as more recent innovations such as complex wavelets [25], steerable pyramids [26], and curvelets [27]) to Poisson data, but wavelets and related representations are easy to use in the Gaussian case. The Haar wavelet system is the only exception [28]; it does provide a tractable multiscale analysis framework for Poisson data, and this paper builds on the Haar-based multiscale likelihood factorizations we developed in [29], [30]. There are several reasons why Gaussian approximations are undesirable. First, the approximations are usually only reasonable if the numbers of detected photons are sufficiently large (so that the Poisson data, possibly after a suitable transformation, is roughly Gaussian distributed). To insure that the photon count levels are large, the detections must be binned or aggregated over regions (usually pixels/voxels) of sufficiently large area/volume. Thus, one must immediately sacrifice spatial resolution in order to accommodate the approximations. This runs counter to the entire philosophy of wavelet and multiscale methods which attempt to achieve some degree of spatial adaptivity in order to recover as much of the image detail and structural nuances as possible from the data. Secondly, to take advantage of the wealth of theoretical, algorithmic, experimental and clinical expertise developed in photon-limited imaging in the past two decades, methods which retain the Poisson likelihood criterion as the fundamental tool for statistical inquiry are quite advantageous. This paper describes new multiscale methods for photon-limited image denoising, deblurring, and reconstruction that are based on the Poisson likelihood and the classical EM algorithm.

Multiscale and wavelet methods fall under the broad heading of computational harmonic analysis (CHA). In image denoising and reconstruction problems, the basic approach pursued by CHA is to (i) define a sufficiently rich class of functions that reasonably captures the characteristics of the images of under study, (ii) find a basis or similar representation consisting of simple, rapidly computable, space-scale localized elements that is capable of approximating all functions in the class with a relatively small number of terms, (iii) employ a coefficient thresholding or 
pruning criterion to remove terms with small (presumably "noisy") coefficients and reconstruct an estimate of the underlying image, and (iv) prove that the estimator is optimal or near optimal in some sense. The basic idea is that because all functions in the class can be represented by a small number of simple elements, it is possible to transform the raw data into the alternate representation (e.g., wavelet) and then remove most of the terms (which in turn eliminates most of the noise) without losing much signal. The result can be a very good estimate of the underlying image. The conventional wisdom is that a representation that provides good approximations will also provide good estimations [18].

This paper introduces a new multiscale image representation based on atoms called platelets. Platelets are localized atoms at various locations, scales and orientations that can produce highly accurate, piecewise linear image approximations to images consisting of smooth regions separated by smooth boundaries. Platelets generalize Donoho's wedgelets [31], and like complex wavelets, steerable pyramids, and curvelets, platelets are capable of concisely representing edges at various scales, locations and orientations. It is shown that platelet approximations can significantly outperform conventional wavelet and wedgelet approximations (in terms of the number of terms required to achieve a given approximation error). Moreover, platelet representations are especially well-suited to the analysis of Poisson data, unlike most other multiscale image representations, and they can be rapidly computed. We propose a platelet-based maximum penalized likelihood criterion that encompasses denoising, deblurring, and tomographic reconstruction problems. The criterion can be incorporated very easily into the classical EM algorithm and the overall computational burden is nearly the same as that of the basic EM-MLE algorithm [5]. This paper is focused on three of the four steps of CHA: (i) defining a class of functions (platelets) suitable for describing the images commonly encountered in medical imaging applications, (ii) studying the approximation capabilities of platelets, (iii) devising MPLEs based on platelets. The performance of platelet-based MPLEs is explored in this paper through a comprehensive set of realistic simulations. Simulation results demonstrate that platelet-based MPLEs can outperform conventional EM-MLE algorithms. The performance of platelet-based MPLEs is also compared 
with the EM-MLE using real data from nuclear medicine and confocal microscopy experiments.

Deriving explicit mathematical bounds on the performance of the MPLEs is beyond the scope of this paper due the the diverse nature of the problems under consideration. However, the impressive approximation capabilities of platelets suggest that the MPLEs may indeed be close to optimal (in a minimax risk sense). Recently, minimax risk bounds for Haar wavelet-based multiscale MPLEs of Poisson intensities have been derived [32] which should be extendable to platelet-based denoising, deblurring and reconstruction. This is currently under investigation and we plan to report on this in a follow-up paper.

The paper is laid out as follows. Section II introduces the platelet representation. A fundamental class of images composed of smooth regions separated by smooth boundaries is defined and it is shown that platelets provide dramatically superior approximations to images in the class compared to Fourier, wavelet and other existing multiscale methods. Section III discusses multiscale analysis methods for Poisson data and the notion of multiscale likelihood factorizations. We show that a Poisson likelihood, parameterized by a platelet representation, admits a multiscale likelihood factorization, which is a probabilistic analog of classical multiresolution analysis. Section IV proposes a new penalized likelihood criterion based on platelets for "denoising" photon-limited images. The multiscale likelihood factorization enables a fast, globally optimal algorithm that computes the MPLE. Section V studies more challenging (inverse) problems including photon-limited image deblurring (restoration) and tomographic reconstruction. It is show that MPLEs can be computed very rapidly using an EM algorithm in which the EStep is identical to that of the classical EM-MLE algorithm and the M-Step is quickly computed using the "denoising" algorithm developed in the previous section. Examples from confocal microscopy are examined through simulation and with real data. Section VI summarizes the new methodology and algorithms and discusses ongoing and future research directions. 


\section{Platelet Decompositions and Image Approximations}

We begin by reviewing Haar multiscale image analysis and its connection to recursive partitions. This sets the stage for developing multiscale platelet representations. The approximation capabilities of platelets are explored and contrasted with Fourier, wavelet, and wedgelet based approximations.

\section{A. Haar Multiscale Analysis and Image Partitioning}

Consider an image $x(u, v)$ on $[0,1] \times[0,1]$. A $J$-scale, Haar multiscale analysis of the image is achieved by defining the dyadic squares

$$
S_{m, n, j} \equiv\left[m / 2^{j},(m+1) / 2^{j}\right) \times\left[n / 2^{j},(n+1) / 2^{j}\right)
$$

for $j=0, \ldots, J-1, m, n=0, \ldots, 2^{j}-1$, where $J$ dictates the size of squares at the finest scale of analysis. Each dyadic square is associated with a coefficient $x_{S_{m, n, j}} \equiv \int_{S_{m, n, j}} x(u, v)$. That is, we define an analysis separating the information in $x$ into its components at various combinations of position and scale $(m, n, j)$. This strategy underlies the analysis of $x$ with respect to an orthonormal basis of dyadic Haar wavelets. Note that each dyadic square $S_{m, n, j}$ splits into four smaller dyadic squares of equal size. These four squares are called the "children" of $S_{m, n, j}$ and are denoted by $\left\{c h\left(S_{m, n, j}^{i}\right)\right\}_{i=1}^{4}$. In the following, the index $i$ will be suppressed to keep the notation cleaner. The coefficients associated with the four children squares, denoted $\left\{x_{c h\left(S_{m, n, j}\right)}\right\}$, will be referred to as the children of $x_{S_{m, n, j}}$.

The relationship between "parent" and children dyadic squares suggests the notion of a recursive partition. The sequence of dyadic squares (from coarse-to-fine) can be interpreted as a recursive dyadic partition of $[0,1]^{2}$. Consider a sequence of nested partitions $\mathcal{P}_{1} \subset \cdots \subset \mathcal{P}_{M}$ of the unit square, where $\mathcal{P}_{1}=[0,1]^{2}, \quad \mathcal{P}_{M}=\cup_{m, n=0}^{2^{J}-1} S_{m, n, J}$, and each partition $\mathcal{P}_{\ell}$ results from splitting one and only one of the dyadic squares in $\mathcal{P}_{\ell-1}$ into four smaller dyadic squares. Hence the collection $\mathcal{P}^{*} \equiv\left\{\mathcal{P}_{\ell}\right\}_{\ell=1}^{M}$ is sometimes called a complete recursive partition $(C-R P)$ of the square $[0,1]^{2}$. 
Note that a C-RP can be associated with a "quad-tree" graph. This tree can be adaptively pruned to produce an incomplete RP with different size squares at different spatial locations. In the dyadic square C-RP, the pruning process is quite similar to thresholding (setting to zero) Haar wavelet coefficients except that the thresholding is performed with a hereditary constraint [33] (i.e., Haar wavelet coefficients at a given scale may be kept only if all "parent" coefficients at coarser scales are retained in the representation). This hereditary constraint ensures that every pruning is a RP with a tree structure; in general, thresholding Haar wavelet coefficients does not correspond to a RP.

Recursive partitions (and hereditary Haar analysis) are interesting because they allow for important extensions of classical Haar multiscale analysis. In particular, one need not restrict the analysis to dyadic square partitions. The wedgelet partition [31] is a dyadic, square recursive partition which allows for non-square, "wedge-shaped" partitions only at the final level of the partition. That is, a wedgelet partition is based on a recursive dyadic square partition of the image in which the final nodes are allowed to terminate with a wedge instead of a square. Consider Figure 1 as a simple illustration of the efficiency of the wedgelet partition space. Figures $1(\mathrm{a})$ and (b) contain rough approximations of the Shepp-Logan phantom to within the same error using the piecewise constant and wedgelet analyses. Notice how many fewer partitions are required for wedgelet approximation.

The wedge split is defined by a line connecting two points on the sides of the square. The points are not arbitrary, rather they are chosen from a finite set of vertices spaced evenly $\delta$ apart around the perimeter of the square. This restriction is crucial because it means that the resulting "dictionary" of wedgelet elements is finite and easily computed. The spacing $\delta$ is referred to as the wedgelet resolution and it is a key parameter of the wedgelet analysis. Following the development in [31], it is assumed that $\delta=2^{-J-K}$ with $K \geq 0$.

The power of wavelets and wedgelets is realized in connection with $m$-term approximations. An m-term approximation to an image is a superposition of any $m$ representation elements (e.g., $m$ wavelet functions or $m$ wedgelets). It is important to note that one can select the $m$ elements 
(a)

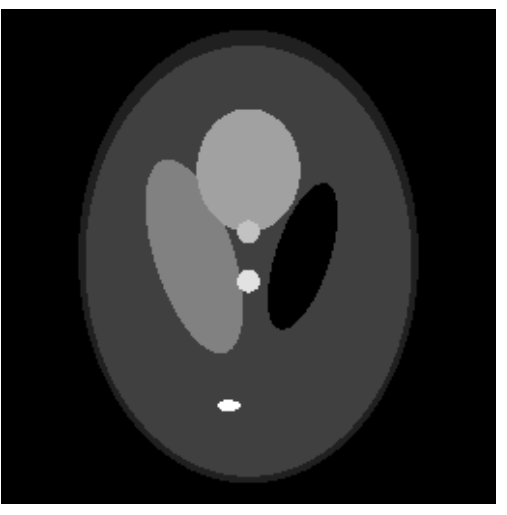

(d)

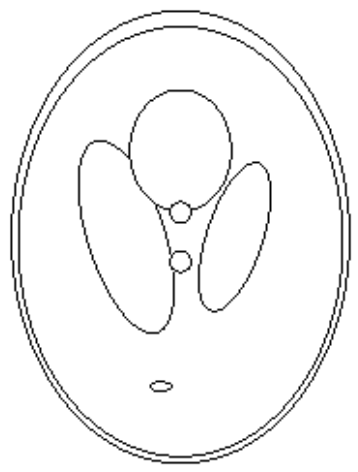

(b)

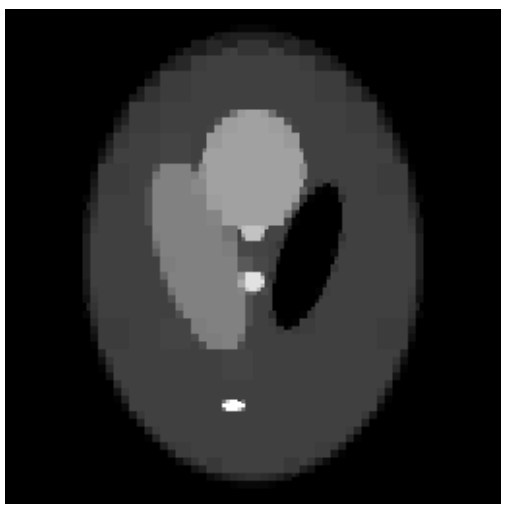

(e)

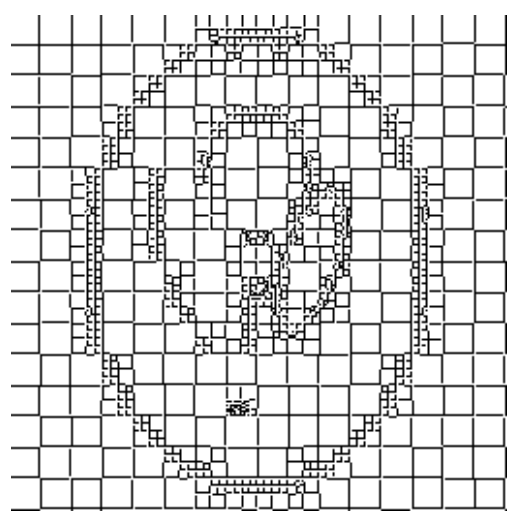

(c)

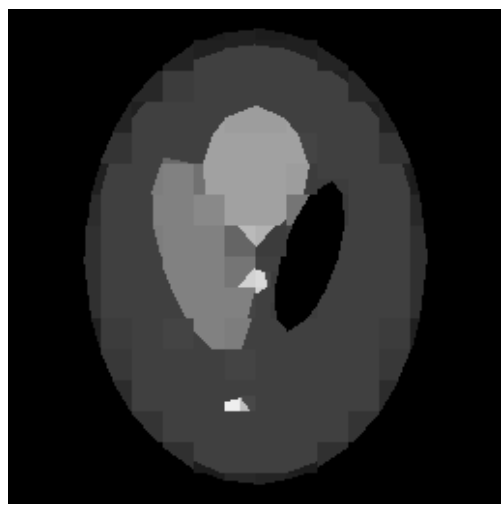

(c)

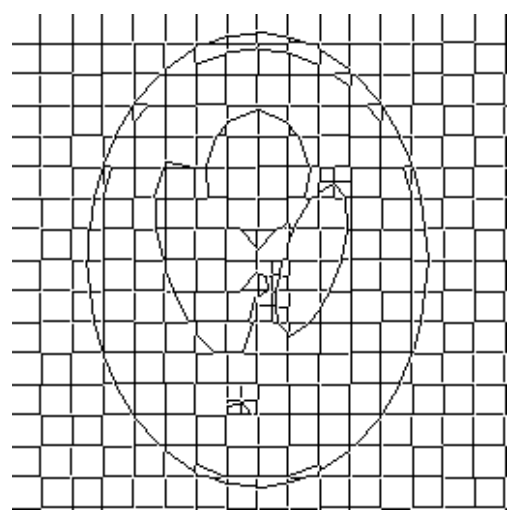

Fig. 1. Partition Spaces (a) Original Image, (b) Constant Approximation (Error $=0.0155$ ), (c) Wedgelet Approximation (Error $=0.0158)$, (d) True Edges, (e) Constant Partition Space, and (f) Wedgelet Partition Space.

that provide the best approximation, where the selection is unconstrained in the case of wavelet approximations and is only constrained by the hereditary conditions dictated by the partition in hereditary Haar and wedgelet approximations. This is sometimes referred to as nonlinear approximation because the selection will depend on the image under consideration (in contrast to linear approximation in which the terms used in the approximation are selected without consideration of the image; e.g., the first (low frequency) $m$-terms in a Fourier series).

\section{B. Platelet Analysis}

Recall that in the standard Haar and wedgelet partitions, the image is modeled as piecewise constant. Instead of approximating the image on each piece of the partition by a constant, we can approximate it with a planar surface. In many applications it is beneficial to have this added 
flexibility. Image gradients, or smooth transitions between regions of varied intensities, encode information about light emission or reflection as well as surface geometry. We define a platelet $f_{S}(x, y)$ to be a function of the form

$$
f_{S}(x, y)=\left(A_{S} x+B_{S} y+C_{S}\right) I_{S}(x, y)
$$

where $A_{S}, B_{S}, C_{S} \in \mathbb{R}, S$ is a dyadic square or wedge associated with a terminal node of a RP, and $I_{S}$ denotes the indicator function on $S$. Each platelet requires three coefficients, compared with the one coefficient for piecewise constant approximation. Although each platelet has two more parameters per term, for images of sufficient smoothness many fewer platelets than constant blocks are needed to approximate an image to within a certain error. Thus, platelet approximations may require far fewer coefficients for a given level of accuracy.

Before proceeding to a more formal analysis of platelet approximation error decay rates, consider the example image in Figure 2. The surface plot of the test image reveals it to be a quadratic "bowl" with a depressed quadratic "bump" in its center. This image provides anecdotal evidence of platelet superiority over piecewise constant and wedgelet approximations.

(a)

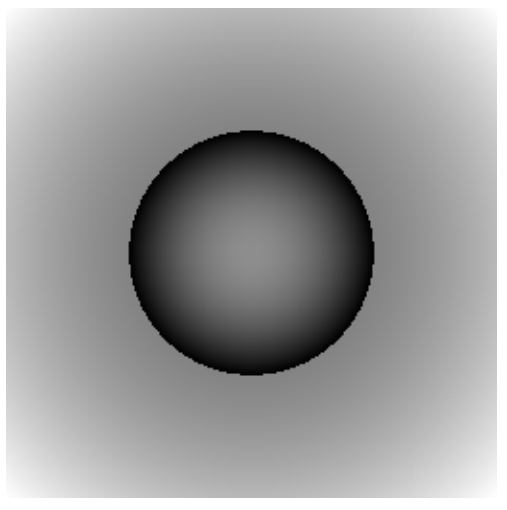

Fig. 2. Quadratic test image. (a) Image. (b) Surface plot.

Approximations with similar $L_{2}$ percent errors were chosen for Figure 3. Figure 3(a) is a hereditary piecewise constant approximation, and contains 2068 constant squares of varying sizes. Figure $3(\mathrm{~b})$ is a wedgelet approximation. Note that this image has an error similar to that of the image obtained with constants, yet it contains only $56 \%$ as many terms, where a 
term is a constant block or a constant wedge; i.e., each block divided into two wedgelets is represented by two terms. Next consider the platelet approximation in Figure 3(c). For each platelet, the coefficients $A_{S}, B_{S}, C_{S}$ are chosen to minimize the squared error of the fit to the image. Again witness comparable approximation error with significantly fewer parameters. Here each dyadic square is fitted with a platelet defined by three coefficients. Finally, Figure 3(d) is an approximation with a platelet fitted to each square or wedge region. In this case a block may be diagonally split into two different gradients. For such a block, we need to store six parameters - three coefficients for each term, or region. In the image below, only 744 parameters are stored, in contrast to the $256 \times 256$ different pixel values in the image. Clearly platelets provide an accurate, sparse representation of smooth images containing smooth boundaries.

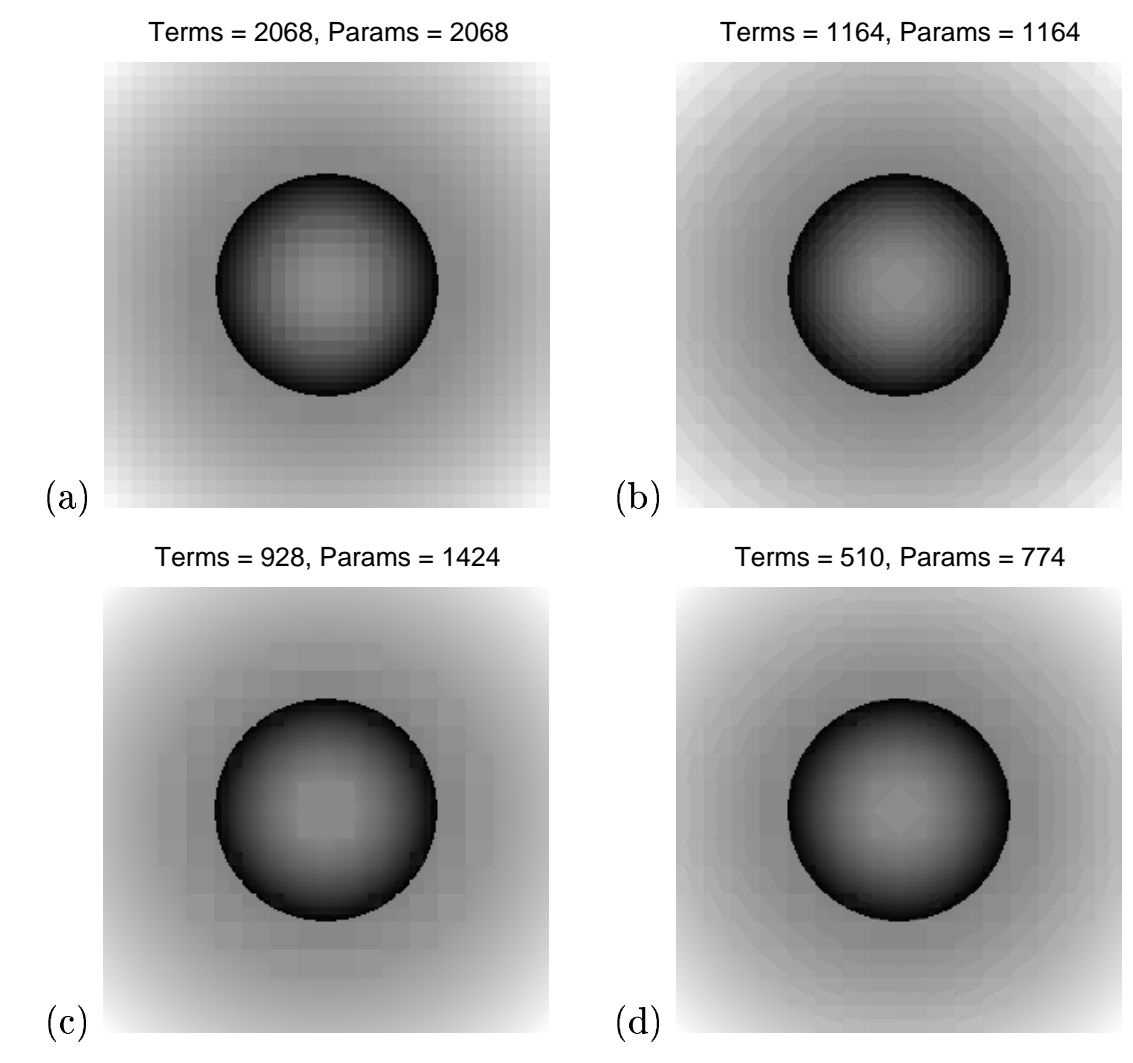

Fig. 3. Approximated Images (a) with constants, (b) with wedgelets, (c) with platelets, (d) and with combined platelets and wedgelets. Each image has error $\approx 3 \times 10^{-4}$.

In fact, Figure 4 shows the decay in approximation error as the number of terms decreases. Not only is the platelet representation more accurate with fewer terms, but also is its rate of decay 
faster than that of the constant and wedgelets representations. What follows is an analytical characterization of these rates.

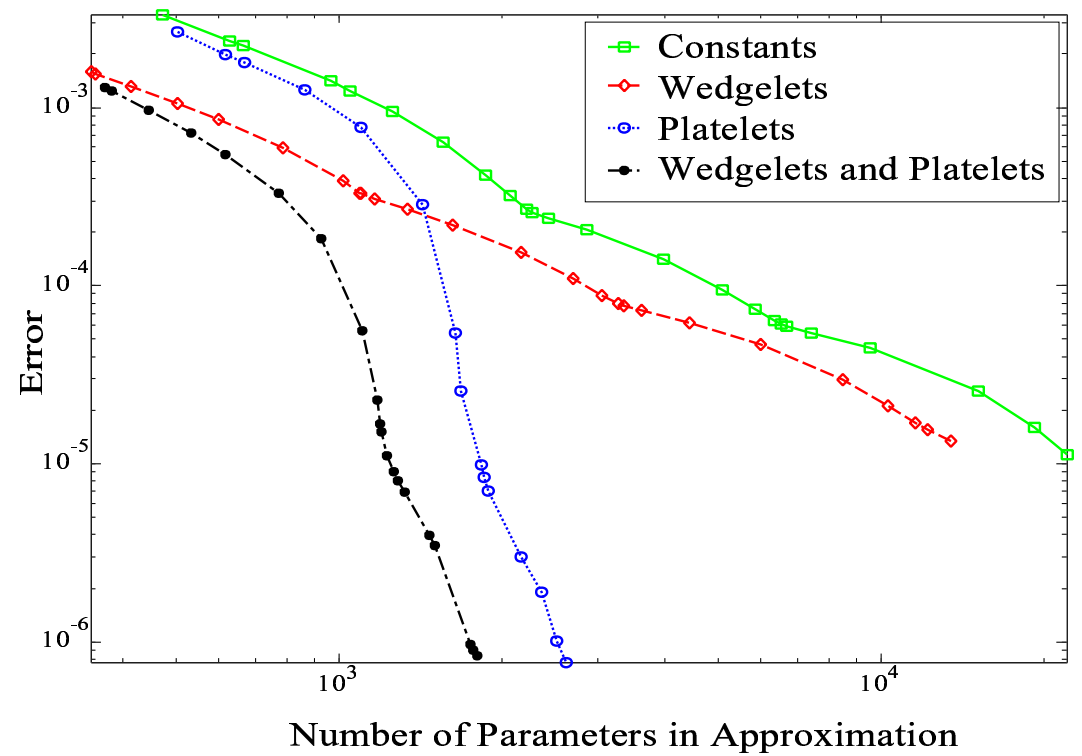

Fig. 4. Approximation errors vs. number of parameters

\section{Platelet Approximation Theory}

For this analysis, consider images which are Hölder smooth apart from a Hölder smooth boundary over $[0,1]^{2}$. Images of this form can be modeled by fusing two (everywhere) smooth images $f_{1}$ and $f_{2}$ into one single image according to

$$
f(x, y)=f_{1}(x, y) \cdot I_{\{y \geq H(x)\}}+f_{2}(x, y) \cdot\left(1-I_{\{y \geq H(x)\}}\right), \quad \forall(x, y) \in[0,1]^{2},
$$

where $I_{\{y \geq H(x)\}}=1$ if $y \geq H(x)$ and 0 otherwise, the function $H(x)$ describes a smooth boundary between a piece of $f_{1}$ and a piece of $f_{2}$. This is a generalization of the "Horizon" image model proposed in [31], which consisted of two constant regions separated by a Hölder smooth boundary.

The boundary is described by $y=H(x)$, where

$$
H \in \operatorname{Hölder}^{\alpha, 1}\left(C_{\alpha}\right), \quad \alpha>1,
$$


where Hölder ${ }^{\alpha, 1}\left(C_{\alpha}\right)$ is the set of functions satisfying

$$
\left|\frac{\partial}{\partial x} H\left(x_{1}\right)-\frac{\partial}{\partial x} H\left(x_{0}\right)\right| \leq C_{\alpha}\left|x_{1}-x_{0}\right|^{\alpha-1}, \text { for all } x_{0}, x_{1} \in[0,1]
$$

The smoothness of the images $f_{1}$ and $f_{2}$ is characterized by a two-dimensional Hölder surface condition

$$
f_{i} \in \operatorname{Hölder}^{\beta, 2}\left(C_{\beta}\right), \quad \beta>1, \quad i=1,2,
$$

where $\operatorname{Hölder}^{\beta, 2}\left(C_{\beta}\right)$ is the set of functions satisfying

$$
\begin{aligned}
\left|\frac{\partial}{\partial x} f_{i}\left(x_{1}, y_{1}\right)-\frac{\partial}{\partial x} f_{i}\left(x_{0}, y_{0}\right)\right| & \leq C_{\beta}\left|\sqrt{\left(x_{1}-x_{0}\right)^{2}+\left(y_{1}-y_{0}\right)^{2}}\right|^{\beta-1} \\
\left|\frac{\partial}{\partial y} f_{i}\left(x_{1}, y_{1}\right)-\frac{\partial}{\partial y} f_{i}\left(x_{0}, y_{0}\right)\right| & \leq C_{\beta}\left|\sqrt{\left(x_{1}-x_{0}\right)^{2}+\left(y_{1}-y_{0}\right)^{2}}\right|^{\beta-1}
\end{aligned}
$$

for all $\left(x_{0}, y_{0}\right),\left(x_{1}, y_{1}\right) \in[0,1]^{2}$. For more information on Hölder spaces see [34].

The model describes a image composed of two Hölder smooth pieces separated by a Hölder smooth boundary. The boundary of the model is specified as a function of $x$ (hence the name "Horizon"), but we could have just as easily specified it as a function of $y$. Furthermore, more complicated boundaries (which are not functions of $x$ or $y$ ) can be constructed with compositions of two or more Horizon-type boundaries.

The squared $L_{2}$ error of $m$-term platelet approximations to images of this form is bounded in the following theorem.

Theorem 1 Consider the class of images

$$
f(x, y)=f_{1}(x, y) \cdot I_{\{y \geq H(x)\}}+f_{2}(x, y) \cdot\left(1-I_{\{y \geq H(x)\}}\right) \quad \forall(x, y) \in[0,1]^{2}
$$

where $f_{i} \in \operatorname{Hölder}^{\beta}\left(C_{\beta}\right), i=1,2$, and $H \in \operatorname{Hölder}^{\alpha}\left(C_{\alpha}\right)$ with $\alpha, \beta>1$. Suppose that $2 \leq m \leq 2^{J}$, with $J>1$. The squared $L_{2}$ error of $m$-term, $J$-scale, resolution $\delta$ platelet approximation to images in this class is less than or equal to $K_{\alpha, \beta} m^{-\min (\alpha, \beta)}+\delta$, where $K_{\alpha, \beta}$ depends on $C_{\alpha}$ and $C_{\beta}$.

Theorem 1 shows that for images consisting of smooth regions $(\beta>1)$ separated by smooth boundaries $(\alpha>1) m$-term platelet approximations may significantly outperform Fourier, wavelet, 
or wedgelet approximations. For example, if the derivatives in the regions and along boundary are Lipschitz ( $\alpha, \beta=2$, i.e., smooth derivatives), then the $m$-term platelet approximation error behaves like $O\left(m^{-2}\right)+\delta$, whereas the corresponding Fourier error behaves like $O\left(m^{-1 / 2}\right)$ and the wavelet and wedgelet errors behave like $O\left(m^{-1}\right)$ at best. For very large $m$, the $\delta$ term will dominate the the platelet approximation error. However, for the modest values of $m$ of the most practical interest the $O\left(\mathrm{~m}^{-2}\right)$ can be most significant and the platelet approximation may then be significantly better than the other approximations. (To more precisely compare the approximation errors one needs to specify the constants $C_{\alpha}$ and $C_{\beta}$, as well as $\delta$.) Wavelets and Fourier approximations do not perform well on this class of images due to the boundary. The reader is referred to [27], [35], [31] for the Fourier and wavelet error rates. Wedgelets can handle boundaries of this type, but produce piecewise constant approximations and perform poorly in the smoother (but non-constant) regions of images.

For the wedgelet case, consider an image of the form $f(x, y)=A x$, a linear gradient image. This image is also in the class under consideration. Wedgelet approximations suffer due to their piecewise constant nature. Because the gradient is constant, the best dyadic $m$-term wedgelet approximation in this case partitions $[0,1]^{2}$ into roughly equal area regions, each of sidelength $O(1 / \sqrt{m})$. The $L_{\infty}$ error in each region is $O(1 / \sqrt{m})$ (because the gradient is a fixed constant). Thus the $L_{2}^{2}$ error in each region is $O\left(m^{-2}\right)$ (squared $L_{\infty}$ error $\times$ area) and the total $L_{2}^{2}$ error of the best $m$-term wedgelet approximation is $O\left(m^{-1}\right)$.

The conclusions of Theorem 1 as well as the error rates of Fourier/wavelet/wedgelet approximations above also carry over to more complicated images composed of several smooth regions and boundaries. Each additional boundary increases the $m$-term approximation errors by integer multiples (two boundaries roughly doubles the error from the case of a single boundary).

\section{Platelet Analysis of Poisson Processes}

Now suppose that $x(u, v)$ is a realization of a Poisson process. Underlying this process is an continuous intensity function $\lambda(u, v),(u, v) \in[0,1]^{2}$. Assume that either by choice or perhaps the 
limitations of measuring instruments, $x(u, v)$ is observed only discretely on the squares (pixels) $S_{m, n}, m, n=0, \ldots, N-1$. It is assumed that the effect of the discretization is to yield an array of count measurements $\boldsymbol{x} \equiv\left\{x_{m, n}\right\}_{m, n=0}^{N-1}$, associated with an array of intensity parameters $\boldsymbol{\lambda} \equiv\left\{\lambda_{m, n}\right\}_{m, n=0}^{N-1}$. Each $x_{m, n}$ is simply the number of events in the square $S_{m, n}$ and $\lambda_{m, n} \equiv$ $\int_{S_{m, n}} \lambda(u, v)$. The counts are conditionally independent; given $\left\{\lambda_{m, n}\right\}, x_{m, n} \sim \operatorname{Poisson}\left(\lambda_{m, n}\right)$. The Poisson likelihood of $\boldsymbol{x}$, given the intensities $\boldsymbol{\lambda}$, is denoted by $p(\boldsymbol{x} \mid \boldsymbol{\lambda})$.

A Haar multiscale analysis of the count data is obtained by associating a count statistic $x_{S_{m, n, j}} \equiv \sum_{k, l:\left(\frac{k}{N}, \frac{l}{N}\right) \in S_{m, n, j}} x_{k, l}$ with each dyadic square $S_{m, n, j}, j=0, \ldots, J-1, m, n=0, \ldots, 2^{j}-$ 1 , and $J=\log _{2}(N)$. There also exists a Haar multiscale analysis of the intensity function which is defined analogously on dyadic squares. In earlier work, we introduced in this context a class of multiscale likelihood factorizations that provide an alternative probabilistic representation (i.e., in addition to that of the original likelihood) of the information in $\boldsymbol{x}$, in a manner indexed by the various position/scale combinations offered by a given $\mathrm{C}-\mathrm{RP} \mathcal{P}^{*}$ (in this case the partition underlying the dyadic Haar analysis) [29], [30], [28]. The keys to the likelihood factorization are the facts that sums of Poisson variates are Poisson and that the conditional distribution of a collection of Poisson variates given their sum is multinomial. Therefore, the conditional distribution of four children $\left\{x_{c h(S)}\right\}$ given the parent $x_{S}$ is multinomial. For a given C-RP $\mathcal{P}^{*}$ the likelihood $p(\boldsymbol{x} \mid \boldsymbol{\lambda})$ is factorized as

$$
p(\boldsymbol{x} \mid \boldsymbol{\lambda})=p\left(x_{S_{0}} \mid \lambda_{S_{0}}\right) \prod_{S \in N T\left(\mathcal{P}^{*}\right)} p\left(\left\{x_{c h(S)}\right\} \mid x_{S}, \theta_{S}\right)
$$

where $S_{0} \equiv[0,1]^{2}$, and $N T\left(\mathcal{P}^{*}\right)$ is the set of all non-terminal squares in $\mathcal{P}^{*}$ (i.e., excluding individual pixels $\left.S_{m, n}\right)$. $\theta_{S}$ denotes the three parameters of the multinomial conditional likelihood of $\left\{x_{c h(S)}\right\}$ given $x_{S}$, which consist of the ratios of the child intensities to the parent intensities. The child intensities are then $\lambda_{S} \theta_{S}^{1}, \lambda_{S} \theta_{S}^{2}, \lambda_{S} \theta_{S}^{3}$, and $\lambda_{S}\left(1-\theta_{S}^{1}-\theta_{S}^{2}-\theta_{S}^{3}\right)$. The expression in (2) serves as a probabilistic analogue of an orthonormal wavelet decomposition of a function. The parameters of the conditional likelihoods play the same role as wavelet coefficients in a conventional wavelet-based multiscale analysis. In fact, there is a one-to-one mapping between 
the multinomial parameters and the Haar wavelet and scaling coefficients. The factorization in (2) can be shown to follow from a set of sufficient conditions whose form and function are remarkably similar to those of a Haar wavelet analysis - effectively a multiresolution analysis of the likelihood function. Details may be found in [28].

For a general (incomplete) RP, say $\mathcal{P}$, certain terminal squares may include several pixels. The multiscale factorization in this general case takes the form

$$
\begin{aligned}
p(\boldsymbol{x} \mid \boldsymbol{\lambda}(\mathcal{P}, \boldsymbol{\theta}))= & p\left(x_{S_{0}} \mid \lambda_{S_{0}}\right) \prod_{S \in N T(\mathcal{P})} p\left(\left\{x_{c h(S)}\right\} \mid x_{S}, \theta_{S}\right) \\
& \times \prod_{c h(S) \in T(\mathcal{P})} p\left(\left\{x_{m, n}\right\}_{(m / N, n / N) \in \operatorname{ch}(S)} \mid x_{c h(S)}\right),
\end{aligned}
$$

where $\boldsymbol{\theta} \equiv \lambda_{S 0} \cup\left\{\theta_{S}\right\}_{S \in(\mathcal{P})}$ and $T(\mathcal{P})$ is the set of all terminal squares in $\mathcal{P}$ and the conditional likelihood corresponding to each terminal square $p\left(\left\{x_{m, n}\right\}_{(m / N, n / N) \in \operatorname{ch}(S)} \mid x_{c h(S)}\right)$ is multinomial with equal probabilities (since the intensity in the square is modeled as constant). Note that in this case the intensity is constrained to be piecewise constant on each piece of the partition, as indicated by the notation $\boldsymbol{\lambda}(\mathcal{P}, \boldsymbol{\theta})$. For readability, however, this function will simply be referred to as $\boldsymbol{\lambda}$ for the remainder of this paper. The fact that a partition of the image space underlies the multiscale likelihood factorization is key. For example, it is not possible to obtain a similar factorization with conventional smooth wavelets. This is simply due to the fact that the distribution of an arbitrary linear combination (e.g., inner product or filtering) of Poisson variables does not have a simple, easily expressed likelihood function (in fact a closed-form expression does not exist). Only unweighted summations of Poisson variables possess a simple expression; namely the sum of independent Poisson variables (e.g., arising when summing over a piece of the partition) is Poisson distributed. Thus, Haar multiscale analysis and its generalizations are especially well suited to the study of Poisson data.

Wedgelet partitions produce a multiscale likelihood factorization of the form (2), in which case the terminal nodes of the partition can denote wedge-shaped regions in addition to squares; that is, $S \in(\mathcal{P})$ may be a dyadic square or wedge. Because the spatial resolution of the acquired image data is limited to pixel size squares $\left(2^{-J} \times 2^{-J}\right)$, the continuous wedgelet partitions discussed in 
the previous section are replaced by "digital" wedgelets. The splitting "line" defining a digital wedgelet is a pixel-scale approximant of the ideal line; i.e., the digital wedgelet split boundary follows the boundaries of pixels, producing a staircase-like approximation to a line. A digital wedgelet splits a dyadic square into two pieces, the two pieces contain disjoint sets of pixels. Note that this also implies that the wedgelet resolution (spacing of vertices) is $\delta=2^{-J}$, the sidelength of a pixel.Additionally, instead of approximating the intensity function on each piece of the partition by a constant, we can approximate it with a planar surface (platelet). The likelihood factorization is still valid, but the terminal node likelihood functions may now be parameterized by a planar intensity function requiring two extra coefficients for the slope of the gradient in addition to a coefficient corresponding to the estimated total intensity of the associated square or wedge shaped region. In this case,

$$
\begin{aligned}
p(\boldsymbol{x} \mid \boldsymbol{\lambda})= & p\left(x_{S_{0}} \mid \lambda_{S_{0}}\right) \prod_{S \in N T(\mathcal{P})} p\left(\left\{x_{c h(S)}\right\} \mid x_{S}, \theta_{S}\right) \\
& \times \prod_{\operatorname{ch}(S) \in T(\mathcal{P})} p\left(\left\{x_{m, n}\right\}_{(m / N, n / N) \in \operatorname{ch}(S)} \mid x_{c h(S)}, \theta_{\operatorname{ch}(S)}\right)
\end{aligned}
$$

where the terminal node likelihood factors $p\left(\left\{x_{m, n}\right\}_{(m / N, n / N) \in \operatorname{ch}(S)} \mid x_{c h(S)}, \theta_{c h(S)}\right)$ are the multinomial likelihoods of the data in $\operatorname{ch}(S)$ given a planar model $\theta_{\operatorname{ch}(S)}$ of the intensity on $\operatorname{ch}(S)$. More specifically, $x_{c h(S)}$ is the total photon count in the region $\operatorname{ch}(S)$. This leaves us with two degrees of freedom (embodied in $\left.\theta_{c h(S)}\right)$ that complete the description of a planar intensity model on $\operatorname{ch}(S) ;$ e.g. $\theta_{\operatorname{ch}(S)}=\left\{A_{\operatorname{ch}(S)}, B_{\operatorname{ch}(S)}\right\}$. It turns out that maximum likelihood estimates of the parameters of the linear surface $\theta_{\operatorname{ch}(S)}$ are easy to compute because, as shown in the next section, the $\log$ likelihood function is concave.

\section{Photon-Limited Image Denoising}

\section{A. Maximum Penalized Likelihood Estimators}

The multiscale likelihood factorizations above provide for a very simple framework for maximum penalized likelihood estimation, wherein the penalization is based on the complexity of the underlying partition. The complexity of a given partition is proportional to the total number 
of regions. For example, a C-RP partitions the image into each individual pixel, and thus has complexity $O\left(N^{2}\right)$. An (incomplete) RP with $R$ regions has complexity $O(R)$. The maximum penalized likelihood criterion we employ here is (recall $\boldsymbol{\lambda}$ is a function of the multiscale parameters and partition; i.e. $\boldsymbol{\lambda}=\boldsymbol{\lambda}(\boldsymbol{\theta}, \mathcal{P}))$

$$
L_{\beta}(\boldsymbol{\lambda}) \equiv \log p(\boldsymbol{x} \mid \boldsymbol{\lambda})-\beta\{\# \boldsymbol{\theta}\}
$$

where $p(\boldsymbol{x} \mid \boldsymbol{\lambda})$ denotes a likelihood (factorization) of the form (2), (3), or (4), and $\{\# \boldsymbol{\theta}\}$ is the number of parameters in the vector $\boldsymbol{\theta}$ (one for each constant region, three for each planar region). The constant $\beta>0$ is a weight that balances between fidelity to the data (likelihood) and complexity regularization (penalty), which effectively controls the bias-variance trade-off.

The solution of

$$
\begin{aligned}
(\widehat{\mathcal{P}}, \widehat{\boldsymbol{\theta}}) & \equiv \underset{\mathcal{P}, \boldsymbol{\theta}}{\arg \max _{\beta}} L_{\beta}(\boldsymbol{\lambda}(\mathcal{P}, \boldsymbol{\theta}) \\
\widehat{\boldsymbol{\lambda}} & \equiv \lambda(\widehat{\mathcal{P}}, \widehat{\boldsymbol{\theta}})
\end{aligned}
$$

is called a maximum penalized likelihood estimator (MPLE). Larger values of $\beta$ produce smoother, less complex estimators; small values of $\beta$ produce more complicated estimators. Note that if $\beta=0$, then no penalty is assigned and a C-RP (with $N^{2}$ free parameters) maximizes the likelihood. Since a C-RP corresponds to a pixel-based partition, in the $\beta=0$ case the MPLE reduces to the conventional maximum likelihood estimator (MLE).

The approximation-theoretic result of Theorem 1 has important implications for the performance of the platelet-based MPLE. The mean square error (MSE) of the MPLE can be separated into squared bias and variance. The variance of the MPLE is proportional to the number of terms $m \propto\{\# \boldsymbol{\theta}\}$ in a platelet approximation. The squared bias is proportional to the error of a $m$ term platelet approximation to the underlying true intensity. We have shown that for a class of images characterized by regions of smooth intensity separated by smooth boundaries platelet approximations with a small number of terms (relative to the number of pixels) can be quite accurate. This means that if the underlying intensity belongs to this class (or if it can be closely approximated by a member of the class), then Theorem 1 shows that for a small value of $m$ there 
exists an accurate $m$-term platelet approximation to the true intensity. If $m \ll N^{2}$, then the reduction in variance will be dramatic, and the platelet-based MPLE will tend to have a small MSE.

Maximizing (5) involves adaptively pruning the C-RP based on the data. This pruning can be performed optimally and very efficiently using bottom-up, CART-like algorithms [33]. A solution to (6) can be computed in $O\left(N^{2}\right)$ operations (i.e., number of operations is proportional to number of pixels), as demonstrated below. The pruning process is akin to a "keep or kill" wavelet thresholding rule. An MPLE provides higher resolution and detail in areas of the image with higher count levels (higher SNR) and/or where strong edges are present. The partition underlying the MPLE is pruned to a coarser scale (lower resolution) in areas with lower count levels (low SNR) and where the data suggest that the intensity is fairly smooth. Moreover, the possibility of wedgelet partitions and planar fits allows the MPLE to adapt to the contours of smooth boundaries in the image and smooth (but non-constant) variation in the image intensity.

\section{B. Optimal Pruning Algorithm}

Observe that the structure of the penalized likelihood criterion stated in (5) and the likelihood factorization described in Section III allow an optimal intensity estimate to be computed quickly. Equation (5) may be expanded using (4) to yield:

$$
\begin{aligned}
L_{\beta}(\boldsymbol{\lambda})= & \log p\left(x_{S_{0}} \mid \lambda_{S_{0}}\right)+\operatorname{pen}\left(\lambda_{S_{0}}\right)+\sum_{S \in N T(\mathcal{P})} \log p\left(\left\{x_{c h(S)}\right\} \mid x_{S}, \theta_{S}\right)+\operatorname{pen}\left(\theta_{S}\right) \\
& +\sum_{c h(S) \in T(\mathcal{P})} \log p\left(\left\{x_{m, n}\right\}_{(m / N, n / N) \in \operatorname{ch}(S)} \mid x_{c h(S)}, \theta_{c h(S)}\right)+\operatorname{pen}\left(\theta_{c h(S)}\right) .
\end{aligned}
$$

The penalty of the total image intensity parameter, $\lambda_{S_{0}}$, is $\beta$ because of the singular dimension of the parameter. Each nonterminal node's penalty, pen $\left(\theta_{S}\right)$, is either $\beta$ or $3 \beta$ because once the value of the parent node is known, there are only one or three free parameters necessary to describe how the intensity is distributed among the children in a wedge split or a quad split, respectively. Finally, each terminal node's penalty, pen $\left(\theta_{\operatorname{ch}(S)}\right)$, is either 0 or $2 \beta$; once the value of the parent node is known, no parameter is needed to represent a constant-valued region, and 
two parameters are needed to to represent the gradient of a planar fit to the data. The likelihood factorization allows the likelihood of the entire image to be represented in a tree structure in which both likelihoods and parameter penalties of children are inherited by parents. Using this, it is possible to optimally prune an RP of the data using a fast algorithm reminiscent of dynamic programming, Coifman and Wickerhauser's "Best-Ortho-Basis" algorithm [36], or the CART algorithm [33].

The goal is to estimate the intensity $\boldsymbol{\theta}$ according to (6). In order to perform the estimation, the algorithm considers each dyadic square in the partition of the observed image and performs an $M$-ary hypothesis test. The hypotheses for each dyadic square are as follows:

- $\mathbf{H}_{\mathbf{0}}$ : Constant $=$ homogeneous square (terminal node)

- $\mathbf{H}_{\mathbf{1}}$ : Wedgelet $=$ two homogeneous wedges (terminal node)

- $\mathbf{H}_{2}$ : Platelet = dyadic linear gradient on square (terminal node)

- $\mathbf{H}_{\mathbf{3}}$ : Wedged Platelet = linear gradients on two wedges (terminal node)

- $\mathbf{H}_{4}$ : Quad split = inherit from children (non-terminal node)

It is also possible to consider of subset of $\left\{\mathbf{H}_{i}\right\}$; e.g. using $\mathbf{H}_{\mathbf{0}}$ and $\mathbf{H}_{\mathbf{4}}$ only coincides with the hereditary Haar analysis (with no wedgelets or platelets). The algorithm begins one scale above the leaf nodes in the quad tree and traverses upwards, performing a tree-pruning operation at each stage. For each node (i.e., dyadic square) at a particular scale, the penalized log likelihoods for each hypothesis are calculated. In particular, the penalized log likelihood for the quad split is computed using the optimal penalized log likelihoods computed at the previous, finer scale for each of the four children. As demonstrated above, these four child penalized log likelihoods add to yield the penalized log likelihood of the parent node, and then this log likelihood is compared with those for the other four hypotheses. If, for a given node, the maximum penalized $\log$ likelihood is associated with a hypothesis other than a quad split, then that node is made a terminal node with parameters appropriate to the said hypothesis; its children are then pruned from the RP. The maximum log likelihood for this newly terminal node will be used for all quad split log likelihood computations for all of the node's ancestors unless it, too, is pruned during 


\begin{tabular}{|ll|}
\hline Initialize: & $j=J-1$ \\
Loop: & for each node $S_{j, m, n}$ at level $j$ \\
Calculate: & $L_{\beta}\left(\boldsymbol{\theta}_{H_{i}} ; S_{j, m, n}\right)$ for $0 \leq i \leq 3$ \\
& $L_{\beta}\left(\boldsymbol{\theta}_{H_{4}} ; S_{j, m, n}\right)=\sum_{S^{\prime} \in \operatorname{ch}\left(S_{j, m, n}\right)} L_{m i n}\left(S^{\prime}\right)$ \\
& $L_{m i n}\left(S_{j, m, n}\right)=\min _{0 \leq i \leq 4} L_{\beta}\left(\boldsymbol{\theta}_{H_{i}} ; S_{j, m, n}\right)$ \\
& $\boldsymbol{\theta}_{m i n}\left(S_{j, m, n}\right)=\arg \min _{0 \leq i \leq 4} L_{\beta}\left(\boldsymbol{\theta}_{H_{i}} ; S_{j, m, n}\right)$ \\
& $j=j-1$ \\
Coarsen Scale: & \\
Goto Loop if $j \geq 0$ & Perform a depth first search for terminal nodes. When a \\
Prune: & terminal node is found, record the MPLE for each pixel de- \\
& scending from the current node.
\end{tabular}

TABLE I

\section{Algorithm Pseudocode}

analysis on a higher scale. The algorithm pseudocode is in Table I. In the table, $L_{\beta}\left(\boldsymbol{\theta}_{H_{i}} ; S_{j, m, n}\right)$ denotes the penalized $\log$ likelihood term for square $S_{j, m, n}$ under hypothesis $H_{i}$.

\section{Computational Complexity}

Before examining the computational complexity of this algorithm, we first establish the concavity of the log likelihood function, which will allow us to perform the optimization rapidly. There is no closed-form solution to the MLE of the plate parameters with respect to the Poisson or multinomial likelihood; however, they can be computed numerically, as described for the Poisson case by Unser [37]. In this case, the likelihood in the plate factorization is concave in the plate parameters, which means that a numerical optimization technique such as Newton's method or gradient descent can find the optimal parameter values. In addition, since there are only two parameters per platelet, the optimization is over a two-dimensional space and may be solved with relatively few iterations of the optimization algorithm of choice. The parameters in 
the case of constant regions or wedgelets are just the count averages, and in that case no such numerical optimization technique is necessary, although the concavity result still holds. The following lemma, proved in Appendix B, establishes the concavity. Consider a terminal node log likelihood term for a platelet $\log p\left(\left\{x_{m, n}\right\}_{(m / N, n / N) \in \operatorname{ch}(S)} \mid x_{c h(S)}, \theta_{\operatorname{ch}(S)}\right)$. The subscripts $\operatorname{ch}(S)$ will be dropped to simplify the notation at this point. The log likelihood is multinomial and the multinomial parameters, denoted $\rho$, are not equal (as in the case of a constant region) but instead obey a linear model of the form $\rho=T \theta$, where $\theta$ is a two-parameter vector describing the gradient of the intensity (and hence the linear relationship between the multinomial parameters $\rho)$ and $T$ is a matrix relating the parameters to the gradient field.

Lemma 1 The log (multinomial) likelihood function of a platelet is concave in $\theta$.

This lemma makes the following bounds of the computational complexity of this algorithm possible. We bound the computational complexity of performing either an "approximate" or exact MPLE, where the "approximate" estimate uses a (suboptimal) least-squares platelet fit and the exact estimate is obtained by numerically optimizing the (concave) log likelihood function. for the most likely platelet fit. The theorem below is also proved in Appendix B.

Theorem 2 (MPLE): A Haar MPLE can be computed in $O\left(N^{2}\right)$ operations, where $N^{2}$ is the number of pixels in the image. A wedgelet or "approximate" platelet MPLE can be computed in $O\left(N^{3}\right)$ operations. An exact platelet $M P L E$ can be computed in $O\left(N^{4}\right)$ operations.

The "approximate" platelet fit is used in all experiments discussed in this paper.

\section{Denoising Experiments and Applications}

Figure 5(a) depicts an image of a heart obtained from a nuclear medicine study. The image was obtained using the radiopharmaceutical Thallium-201. In this type of study, the radiopharmaceutical is injected into the bloodstream of the patient and moves into the heart wall in proportion to the local degree of blood perfusion. The purpose of the procedure is to determine if there is decreased blood flow to the heart muscle. Figure 5(f) depicts an image of the spine 
obtained from a nuclear medicine study. The radiopharmaceutical used here is Technetium- $99 \mathrm{~m}$ labeled diphosphonate. In bone studies such as this, brighter areas indicate increased uptake of blood in areas where bone growth or repair is occurring. Functional changes in the bone can be detected using nuclear medicine image before they will show up in X-ray images. More information on this data can be found in [38].

The hereditary Haar and platelet based MPLEs are shown below below. The first set of estimates (Figures 5 (b),(d),(g), and (i)) were generated by one pass through the denoising algorithm. For the second set (Figures $5(\mathrm{c}),(\mathrm{e}),(\mathrm{h})$, and $(\mathrm{j})$ ), we applied a technique called "averaging over shifts" or "cycle-spinning" [39], [40]. This entails circularly shifting the raw data by a few pixels, denoising, and then shifting the estimate back to its original position. Five shifts in each direction (horizontal and vertical) yielded a total of twenty-five estimates, which were then averaged. This technique is often improves denoising and reconstruction results because it reduces the dependence on the dyadic partition. We employ this technique in all subsequent experiments.

\section{Photon-Limited Image Restoration and Reconstruction}

In many medical imaging applications, the detected photons are indirectly related to the object of interest. For example, confocal imaging systems may involve a blurring process, and SPECT and PET require the measurement of tomographic projections. Blurring and tomographic projections can be described statistically as follows. Photons are emitted (from the emission space) according to an intensity $\boldsymbol{\lambda}$. Those photons emitted from location $(k, l)$ are detected (in the detection space) at position $(m, n)$ with transition probability $p_{k, l, m, n}$. In such cases, the measured data are distributed according to

$$
x_{m, n} \sim \text { Poisson }\left(\sum_{k, l} p_{k, l, m, n} \lambda_{k, l}\right) .
$$

Notice that the intensity of the observation is $\sum_{k, l} p_{k, l, m, n} \lambda_{k, l}$, rather than $\lambda_{m, n}$ as in the direct case. The transition probabilities $p_{k, l, m, n}$ represent the blurring or projection process. The recovery $\boldsymbol{\lambda}$ from $\boldsymbol{x}$ is an inverse problem; one must invert the effects of $\boldsymbol{p} \equiv\left\{p_{k, l, m, n}\right\}$. 
The Poisson likelihood function of $\boldsymbol{x}$ given $\boldsymbol{\lambda}$ is denoted $p(\boldsymbol{x} \mid \boldsymbol{\lambda})$. The log-likelihood is

$$
\log p(\boldsymbol{x} \mid \boldsymbol{\lambda})=\sum_{m=0}^{M_{1}} \sum_{n=0}^{M_{2}}\left(-\sum_{k, l=0}^{N-1} p_{k, l, m, n} \lambda_{k, l}+x_{m, n} \log \left(\sum_{k, l=0}^{N-1} p_{k, l, m, n} \lambda_{k, l}\right)-\log x_{m, n} !\right),
$$

where $M_{1} \times M_{2}$ is the dimension of the detection space and $N \times N$ is the dimension of the emission (or image) space. $M_{1}$ and $M_{2}$ are arbitrary, but for convenience assume that $N$ is a power of two. The likelihood function here is much more complicated due the the presence of $\boldsymbol{p}$, and a multiscale likelihood factorization is not possible. Therefore the inverse problem faced in deblurring (restoration) and tomographic reconstruction cannot be solved by simple tree-pruning methods. The multiscale approach developed in the previous chapters can however be applied within the context of an EM algorithm. The key idea in the EM algorithm (as it generally applies to photon-limited imaging problems) is that the indirect (inverse) problem can be broken into two subproblems; one which involves computing the expectation of the unobserved direct data (as though no blurring or projection took place) and one which entails estimating the underlying image from this expectation.

Central to the EM algorithm is the notion of "complete" data [16] defined as $\boldsymbol{z}=\left\{z_{k, l, m, n}\right\}$, where $z_{k, l, m, n}$ denotes the number of photons emitted from $(k, l)$ and detected at $(m, n)$. The complete data are Poisson distributed according to

$$
z_{k, l, m, n} \sim \operatorname{Poisson}\left(\lambda_{k, l} p_{k, l, m, n}\right)
$$

Hence the observed data $\boldsymbol{x}$ in (V) are given by $x_{m, n}=\sum_{k, l} z_{k, l, m, n}$. Additionally, were we able to observe $\boldsymbol{z}=\left\{z_{k, l, m, n}\right\}$, the direct emission data for each location $(k, l)$ is given by sums of the form $y_{k, l} \equiv \sum_{m, n} z_{k, l, m, n}$, from which it follows that $y_{k, l} \sim \operatorname{Poisson}\left(\lambda_{k, l}\right)$. Therefore, if $\boldsymbol{z}$ were known, we could avoid the inverse problem altogether and simply deal with the issue of estimating a Poisson intensity given direct observations.

\section{A. Maximum Likelihood Estimation}

It is well-known that the maximizer of (7) cannot be expressed in closed-form, but the concavity of the log-likelihood allows a numerical determination. While in principle any numerical 
optimization method could be used, the iterative EM algorithm, as first proposed for this problem in [41], has a number of features that make it especially desirable, most notably its natural, probabilistic formulation, computationally straightforward calculations at each iteration step, and numerical stability [16]. Moreover, it can be shown that the EM algorithm monotonically increases the log-likelihood at each iteration and converges to a global (not necessarily unique) point of maximum for (7) [5].

Unfortunately, due to the ill-posed nature of the likelihood equations, the variance of the MLE can be quite high, particularly for applications involving low counts. In fact, in many cases the MLE is practically useless. A popular remedy is to stop the EM algorithm prior to convergence (e.g., [17]). Stopping the algorithm acts implicitly as a smoothing operation and can produce acceptable results. However, it may be preferable to abandon the strictly likelihoodbased perspective altogether, and approach the inverse problem with a different criterion, one that smoothes through a well-defined optimal solution, while still providing useful and meaningful results.

\section{B. MPLE using Platelet Approximations}

The maximum penalized likelihood function employed here is

$$
L_{\beta}(\boldsymbol{\lambda}) \equiv \log p(\boldsymbol{x} \mid \boldsymbol{\lambda})-\beta\{\# \boldsymbol{\theta}\}
$$

where $\{\# \boldsymbol{\theta}\}$ is the number of parameters in the vector $\boldsymbol{\theta}$. Again, keep in mind that the intensity is a function of the partition and the multiscale parameters; i.e., $\boldsymbol{\lambda}=\boldsymbol{\lambda}(\boldsymbol{\theta}, \mathcal{P})$. The constant $\beta>0$ is again weight that balances between fidelity to the data (likelihood) and complexity penalty. In maximizing this function, the resulting reconstruction will be one that has a relatively high likelihood value as well as a relatively low complexity Haar, wedgelet, or platelet representation. The EM algorithm can be easily modified to produce a sequence of reconstructions that monotonically increase this function. To simplify the notation in the derivation of the EM algorithm, the intensity functions will be denote by $\boldsymbol{\lambda}$ (without explicit indication of the dependence on $\mathcal{P}$ and $\boldsymbol{\theta}$ ). 
The EM algorithm is based on consideration of the following alternative or surrogate function:

$$
L_{\beta}^{c}(\boldsymbol{\lambda}) \equiv \log p(\boldsymbol{z} \mid \boldsymbol{\lambda})-\beta\{\# \boldsymbol{\theta}\}
$$

where the likelihood of the observed data $\boldsymbol{x}$ is replaced by the likelihood of the (unobserved) complete data $z$. The E-Step of the algorithm computes the conditional expectation of $L_{\beta}^{c}(\boldsymbol{\lambda})$ given the observed data. This expectation is computed using the Poisson distribution corresponding to the previous iterate of the algorithm. That is, the E-Step at the $i+1$-th iteration computes

$$
Q\left(\boldsymbol{\lambda}^{(i)}, \boldsymbol{\lambda}\right) \equiv E_{\boldsymbol{\lambda}^{(i)}}\left[L_{\beta}^{c}(\boldsymbol{\lambda}) \mid \boldsymbol{x}\right]
$$

where the subscript on the expectation is the previous iterate $\boldsymbol{\lambda}^{(i)}$ of the algorithm and indicates that the expectation is computed using the Poisson distribution of that intensity. Note that the conditional expectation is

$$
E_{\boldsymbol{\lambda}^{(i)}}\left[L_{\beta}^{c}(\boldsymbol{\lambda}) \mid \boldsymbol{x}\right]=E_{\boldsymbol{\lambda}^{(i)}}[\log p(\boldsymbol{z} \mid \boldsymbol{\lambda}) \mid \boldsymbol{x}]-\beta\{\# \boldsymbol{\theta}\}
$$

The penalty term does not depend on $\boldsymbol{x}$ and so it is simply a constant in this step. Therefore, the E-Step here is equivalent to the E-Step of the conventional EM-MLE algorithm; it computes $E_{\boldsymbol{\lambda}^{(i)}}[\log p(\boldsymbol{z} \mid \boldsymbol{\lambda}) \mid \boldsymbol{x}]$. The complete data $\log$ likelihood $\log p(\boldsymbol{z} \mid \boldsymbol{\lambda})$ happens to be a linear function of $\boldsymbol{z}$ and so this calculation simplifies to computing the $\boldsymbol{z}^{(i)} \equiv E_{\boldsymbol{\lambda}^{(i)}}[\boldsymbol{z} \mid \boldsymbol{x}]$. A closed-form expression for this calculation can be found in [5]. In general each E-Step requires $O\left(M_{1} M_{2} N^{2}\right)$ operations, but often the structure of $\boldsymbol{p}$ can be exploited to simplify the calculation (e.g., if $\boldsymbol{p}$ corresponds to a convolution and $M_{1}=M_{2}=N$, then the E-Step can be calculated in $O\left(N^{2}\right)$ operations).

The M-Step of the algorithm is the maximization of $Q\left(\boldsymbol{\lambda}^{(i)}, \boldsymbol{\lambda}\right)$ over $\boldsymbol{\lambda}$. The penalty, which is a function of $\boldsymbol{\lambda}$, plays a key role in this step. Based on the derivation of the EM algorithm in [42] it can be

$$
Q\left(\boldsymbol{\lambda}^{(i)}, \boldsymbol{\lambda}\right)=\log p\left(\boldsymbol{y}^{(i)} \mid \boldsymbol{\lambda}\right)-\beta\{\# \boldsymbol{\theta}\}+C(\boldsymbol{x})
$$

where $\boldsymbol{y}^{(i)}$ is the unobserved direct data (computed from $\boldsymbol{z}^{(i)}$ ) and $C(\boldsymbol{x})$ is a constant depending on $\boldsymbol{x}$ but not $\boldsymbol{\lambda}$. Thus, the M-Step is equivalent to maximizing $\log p\left(\boldsymbol{y}^{(i)} \mid \boldsymbol{\lambda}\right)-\beta\{\# \boldsymbol{\theta}\}$. Re- 
markably, because $\boldsymbol{y}$ is the direct data, the M-Step is equivalent to the denoising calculation discussed in the previous section with $\boldsymbol{y}^{(i)}$ in place of $\boldsymbol{x}$. To compute the M-Step, first compute $y_{k, l}^{(i)}=\sum_{m, n} z_{k, l, m, n}^{(i)}$, where $z^{(i)}$ is computed in the E-Step. Then carry out the pruning algorithm described in the previous section to compute the vector $\boldsymbol{\theta}$ (and from $\boldsymbol{\theta}$ construct $\boldsymbol{\lambda}$ ) that maximizes $Q\left(\boldsymbol{\lambda}^{(i)}, \boldsymbol{\lambda}\right)$. The result the next iterate of the algorithm, $\boldsymbol{\lambda}^{(i+1)}$.

The EM algorithm must be initialized, and in all examples considered here the initialization is a constant image of total intensity equal to the total number of counts detected. The penalized $\log$ likelihood of each iterate is equal to or greater than that of the previous iterate (monotonicity of EM), however the algorithm is not guaranteed to converge to a global maximum of (9). Two applications of the MPLE and EM algorithm are examined next.

\section{Confocal Microscopy}

Confocal microscopy is used to obtain volume images of small fluorescent objects with high spatial resolution [3]. To generate a confocal fluorescence microscope (CFM) image, the microscope performs a $3 \mathrm{D}$ scan of the object. At each point in the scan, a photo multiplier tube measures the emission of fluorescence light from the object, essentially acting as a photon counter.

Due to the geometry of these microscopes, a "blurring" is introduced into the measurement process. This distortion of the image is commonly modeled by the convolution of the true image with the point spread function of the microscope. Since the arrival of fluorescence light at the photo multiplier tube can be modeled as a Poisson process, the "de-blurring" and estimation process may be viewed as a Poisson inverse problem well suited to the application of iterative estimation using the EM algorithm, as detailed in [6]. The E-step of the algorithm is rapidly computed using the FFT to perform the convolution. In practice, the M-step commonly consists of performing maximum likelihood estimation, but these estimates are known to diverge from the true image after several iterations. A common use for CFMs is the imaging the dendritic spines of neurons. In order to demonstrate the capabilities of the platelet-based MPLE in confocal microscopy, we have created a scale phantom of an image of a dendrite segment with thin, 
stubby, and mushroom spines. See [43] for descriptions of dendrites and the various types of spines. Some regions of the object will be closer to the detector or have more fluorescence than others, resulting in image gradients and making this an excellent candidate for platelet analysis.

Figure $6(\mathrm{a})$ contains the $128 \times 128$ phantom developed for this experiment, and Figure $6(\mathrm{~d})$ contains the blurred and noisy data. The best MLE estimate in Figure 6(b) is the MLE image at the iteration when its $L_{2}$ error was smallest. In practice it is not possible to know which iteration yielded the best ML estimate, but it is included here to demonstrate that MPLE algorithms converge to a point with lower error than the best possible image obtainable with the commonly used stopped-MLE technique [17]. Figure 6(c) is a closeup of a small region of Figure 6(b). The averaged-over-shifts Haar-based MPLE appears in Figure 6(e), and its closeup in Figure 6(f). Likewise, the averaged-over-shifts platelet-based MPLE appears in Figure 6(h) and its closeup in Figure 6(i). Finally, Figure 6(g) plots the $L_{2}$ error of each of the three estimates at each iteration. After several iterations the EM-ML estimate worsens considerably with each subsequent iteration. In contrast, both MPLEs converge eliminating the need to choose which iteration is the best stopping point, as done with the EM-MLE. Furthermore, the converged MPLEs exhibit significantly less $L_{2}$ error than the best MLE.

Figure 7 demonstrates the capabilities of platelet analysis on a real confocal microscopy image of a dendritic spine. In this case the structure of the dendritic spines is of critical interest to researchers. As demonstrated, the platelet-based MPLE can perform better than the stopped MLE.

\section{Emission Computed Tomography}

Here we consider the application of our framework to emission computed tomography (ECT). In medical ECT, a human subject is injected with a radioactive pharmaceutical specifically designed for absorption in certain bodily organs or tissues. The distribution of this pharmaceutical within the subject can provide functional and/or anatomical diagnostic information. To obtain a mapping of pharmaceutical uptake, data are collected by detecting gamma-ray photons that are 
emitted from within the subject as the pharmaceutical decays. From these projection data (the indirect data $\boldsymbol{y}$ in our problem), we wish to estimate the underlying pharmaceutical distribution (intensity $\boldsymbol{\lambda}$ ). The probability transition matrix $\boldsymbol{p}$ is derived from the physics and geometry of the detection device and data collection process [5].

In Figure 8 we illustrate the application of our multiscale framework to a simulated single photon ECT problem. The underlying 2-d intensity in our simulation is the common SheppLogan model, a standard benchmark in SPECT. The intensity $\boldsymbol{\lambda}$ is a $64 \times 64$ square image shown in Figure 8(a). The transition probability matrix $\boldsymbol{p}$, corresponding to a parallel strip-integral geometry with 80 radial samples and 60 angular samples distributed uniformly over $180^{\circ}$, was generated by the ASPIRE software system [44]. $\boldsymbol{p}$ was applied to $\boldsymbol{\lambda}$ to obtain $\boldsymbol{\mu}$, and we used a standard Poisson random number generator to synthesize the projection data $\boldsymbol{y}$. The SheppLogan model is piecewise constant, and so we employ a wedgelet-based MPLE instead of using platelets.

For comparison, in Figure 8(b) we show the very best likelihood-based reconstruction obtained by stopping the likelihood-based EM algorithm at the reconstruction having the smallest squared error, which is impossible to determine in practice since the true intensity is unknown. The Haar and wedgelet based MPLE algorithms converge to good reconstructions, better in quality than the best possible reconstruction obtained by the stopped likelihood-based EM algorithm. The wedgelet based MPLE performs best overall. Note that, unlike the EM-MLE algorithm for which the error diverges, the MPLE errors settle down towards minimums as we continue to iterate.

\section{Conclusions And Ongoing Work}

This paper introduced the platelet representation for the analysis, denoising, and reconstruction of photon-limited medical images. Platelets outperform conventional wavelet representations because of their ability approximate smooth boundaries more efficiently than wavelets. Moreover, because the platelet analysis of Poisson distributed images is tractable and computationally efficient, existing image deblurring and tomographic reconstruction methods based 
on expectation-maximization algorithms can be easily enhanced with platelet-based complexity penalties with only a modest increase in computational complexity. Experimental results with real and simulated data from confocal microscopy and nuclear medicine demonstrate that platelet-based methods can outperform the popular and widely used EM-MLE method.

We feel that the platelet-based MPLE has an important theoretical strength over more conventional approaches like the stopped EM-MLE and methods based on quadratic or non-quadratic roughness penalties or smoothness priors. As discussed in Section IV, because platelets can accurately approximate images with a very small number of terms the platelet-based MPLE can have a very small bias (approximation error) and variance (proportional to the number of terms in the platelet representation). Although the conventional methods mentioned above have been studied extensively with simulated and real data experiments and have been demonstrated to provide high quality image reconstructions, we are not aware of any comparable theoretical results for these methods. Certainly, very little is known about the theoretical error performance of MPLEs based on standard quadratic or even non-quadratic edge preserving roughness penalties for the class of images we considered in our platelet analysis. Even less is known for the stopped EM approaches. It may be possible to compare MPLEs using quadratic penalties with those based on platelets. Quadratic penalties can be interpreted as a penalty weighting applied to a Fourier expansion of the intensity. We have demonstrated that platelet approximations to piecewise smooth intensity functions can significantly outperform Fourier approximations. Thus, we may expect that the error performance of the platelet-based MPLE will be considerably better than that of quadratically penalized MPLEs.

In contrast, it should be possible to quantify the theoretical error performance of the plateletbased MPLE very precisely. In [32] we show that the Haar-based MPLE is near minimax optimal when the underlying Poisson intensity belongs to Bounded Variation or Besov function spaces [34]. These spaces are characterized by mostly smooth images with isolated point singularities. Our interest in this work is in images with singularities along smooth curves (edges and boundaries) rather than at points, and we expect that improved minimax bounds can be obtained for 
our platelet-based MPLE in such cases. We are currently pursuing this work. Minimax error bounds should be obtained relatively easily in the denoising context (applications without a blurring or projection operator). Inverse problems like the tomography case are more challenging to analyze, but in related problems with Gaussian noise, wavelet-based approaches have been shown to be near minimax optimal [45]. We are investigating similar approaches to the Poisson deblurring and tomography problems examined in this paper. The platelet approximation results of this paper are a key first step in this direction.

In addition to the ongoing theoretical analysis of the platelet-based MPLE, experimental comparisons between it and conventional methods are necessary. Furthermore, platelet analysis can be extended to medical imaging problems involving other noise distributions. The multiscale likelihood factorizations that underpin the platelet-based MPLE also exist for Gaussian, multinomial and other data types [28] and only difference in the platelet analysis in these cases will be the parametric form of the conditional likelihood factors involved. In fact, in the Gaussian case all the conditional likelihood factors are Gaussian themselves and the maximum likelihood criterion is equivalent to the conventional least squares criterion. The multinomial case is identical to the Poisson factorization except that the total intensity is fixed to one.

\section{APPENDIX}

\section{Proof of Approximation Theorem}

Proof of Theorem 1 It suffices to verify the theorem for dyadic $m$ (powers of two); an error bound in the general case follows in a similar manner. The proof is constructive. For the most part, we will not specify the constants underlying the bounds discussed below and only speak in terms of orders of magnitude. It is possible to keep careful track of the constants, but this would make the proof less transparent.

First consider approximating the boundary with a $J$-scale (i.e., dyadic squares have sidelength greater than or equal to $2^{-J}$ ) wedgelet partition. From [31] it is known that one can construct an $O(m)$-term dyadic wedgelet partition such that the boundary is completely contained in disjoint 
dyadic squares of equal sidelength $1 / m$. To sketch the idea, imagine tiling $[0,1]^{2}$ with $m^{2}$ dyadic squares of sidelength $1 / m$. Because the boundary function is $\operatorname{Hölder}^{\alpha, 1}\left(C_{\alpha}\right)$, with $\alpha>1$, it must also be Lipshitz (i.e., Hölder ${ }^{1,1}\left(C_{1}\right)$, where the Lipschitz constant $C_{1}$ may be smaller than $C_{\alpha}$ ) it is easy to check that the boundary passes through only $O(m)$ of these squares. Merge all squares not containing the boundary into larger dyadic squares (according to the RP associated with Haar analysis). It turns out that after merging there are only $O(m)$ squares in total. That is, there exists a constant $C>0$ such that the total number of squares is less than or equal to $C m$. It can be shown that $C^{\prime}=8\left(C_{1}+2\right)$ will work [31], where $C_{1}$ is the Lipschitz constant above. This result holds for $2 \leq m \leq 2^{J}$.

Now consider the approximation of a wedgelet to the true boundary in one of the dyadic squares above. Each such square can be broken into three regions, two regions in which the true boundary and wedge boundary agree and one region where they disagree (area "between" the true boundary and the wedge boundary). The area between the true boundary and the wedgelet boundary in a square is $O\left(m^{-(\alpha+1)}\right)$ at most. This is a simple consequence of the fact that the $\operatorname{Hölder}^{\alpha}\left(C_{\alpha}\right)$ can be used to bound the $L_{\infty}$ approximation error between the boundary function $H(x)$ and a line connecting the points $(i / m, H(i / m))$ and $((i+1) / m, H((i+1) / m))$, where $i / m$ and $(i+1) / m$ refer to the horizontal boundaries of a given square. The $L_{\infty}$ error bounds the area of the region in question using this line fit; the error is $O\left(m^{-(\alpha+1)}\right)$. Wedgelets do not use arbitrary vertices (e.g., $H(i / m))$, but rather their vertices are restricted to equispaced points $\delta$ apart along the boundary of each square. This "quantization" effect adds an additional amount of area to the region. This additional amount is $O\left(\delta m^{-1}\right)$.

Next generate a new partition by subdividing all squares of sidelength larger than $1 / \sqrt{m}$ in the $O(m)$ wedgelet partition so that the new partition is a tiling of $[0,1]^{2}$ with dyadic squares having sidelength $1 / \sqrt{m}$ or less. This partition also has $O(m)$ regions. To see this, note that tiling $[0,1]^{2}$ with squares of sidelength $1 / \sqrt{m}$ requires $m$ such squares, so the additional subdivision adds less than $m$ additional squares to the original $O(m)$ regions in the wedgelet partition. The $L_{2}^{2}$ error on each square of the partition just constructed above can be upper bounded as follows. 
First consider squares not containing the boundary (i.e., squares in smooth parts of the image). The first-order Taylor series expansion of $f$ on such a square is

$$
\widehat{f}(x, y)=f\left(x_{o}, y_{o}\right)+\left(x-x_{o}\right) \frac{\partial f}{\partial x}\left(x_{o}, y_{o}\right)+\left(y-y_{o}\right) \frac{\partial f}{\partial y}\left(x_{o}, y_{o}\right)
$$

This expansion provides a planar approximation to $f$ on that square. Because each square has sidelength of at most $1 / \sqrt{m}$, The mean value theorem allows us to bound the the approximation error at any point $(x, y)$ in the square:

$$
\begin{aligned}
|f(x, y)-\widehat{f}(x, y)|= & \mid f\left(x_{o}, y_{o}\right)+\left(x-x_{o}\right) \frac{\partial f}{\partial x}\left(x^{\prime}, y^{\prime}\right)+\left(y-y_{o}\right) \frac{\partial f}{\partial y}\left(x^{\prime}, y^{\prime}\right) \\
& -f\left(x_{o}, y_{o}\right)-\left(x-x_{o}\right) \frac{\partial f}{\partial x}\left(x_{o}, y_{o}\right)-\left(y-y_{o}\right) \frac{\partial f}{\partial y}\left(x_{o}, y_{o}\right) \mid \\
\leq & \left|\left(x-x_{o}\right)\left[\frac{\partial f}{\partial x}\left(x^{\prime}, y^{\prime}\right)-\frac{\partial f}{\partial x}\left(x_{o}, y_{o}\right)\right]\right|+\left|\left(y-y_{o}\right)\left[\frac{\partial f}{\partial y}\left(x^{\prime}, y^{\prime}\right)-\frac{\partial f}{\partial y}\left(x_{o}, y_{o}\right)\right]\right|
\end{aligned}
$$

where $x^{\prime}\left(y^{\prime}\right)$ is between $x$ and $x_{0}\left(y\right.$ and $\left.y_{0}\right)$. From here, the $\operatorname{Hölder}^{\beta}\left(C_{\beta}\right)$ assumption gives the upper bound

$$
\begin{aligned}
|f(x, y)-\widehat{f}(x, y)| & \leq C_{\beta}\left\|\sqrt{\left(x^{\prime}-x_{o}\right)^{2}+\left(y^{\prime}-y_{o}\right)^{2}}\right\|^{\beta-1}\left(\left|x-x_{o}\right|+\left|y-y_{o}\right|\right) \\
& \leq C_{\beta}\left(\frac{\sqrt{2}}{\sqrt{m}}\right)^{\beta-1} \cdot \frac{2}{\sqrt{m}} \\
& =O\left(m^{-\beta / 2}\right)
\end{aligned}
$$

Since this holds for all $(x, y)$ in the square, we have bounded the $L_{\infty}$ error. Thus the $L_{2}^{2}$ error over each such square is $O\left(m^{-(\beta+1)}\right)$ (i.e., $L_{\infty}$ error squared $\times$ area).

Next consider squares containing the boundary. Each such square has a sidelength of $1 / \mathrm{m}$. According to the wedgelet analysis above, the area between the true boundary and the wedge boundary in each square is $O\left(m^{-(\alpha+1)}\right)$. (Since the image is $\operatorname{Hölder}^{\beta, 2}\left(C_{\beta}\right), \beta>1$, it is also Lipschitz and hence continuous and since the image has compact support, it must be bounded and therefore the $L_{2}^{2}$ error in this region is $\left.O\left(m^{-(\alpha+1)}+\delta m^{-1}\right)\right)$. Select planar fits on the two wedges so that the $L_{2}^{2}$ errors in the other two smooth regions of the square are $O\left(m^{-2(\beta+1)}\right)$ (the 2 in the exponent appears because the sidelength is $O(1 / m)$ instead of $O(1 / \sqrt{m})$ ). This gives a total $L_{2}^{2}$ error in each such square of $O\left(m^{-\min (\alpha+1,2(\beta+1))}+\delta m^{-1}\right)$. Combining the planar 
approximations on dyadic squares of sidelength $1 / \sqrt{m}$ or less in the smooth parts of the image with the platelet approximation of the boundary on dyadic squares of sidelength $1 / \mathrm{m}$ produces a total $L_{2}^{2}$ approximation error of $O\left(m^{-\min (\alpha, \beta)}+\delta\right)$. That is, the squared $L_{2}$ approximation error is bounded above by $K_{\alpha, \beta}\left(m^{-\min (\alpha, \beta)}+\delta\right)$, where the constant $K_{\alpha, \beta}$ depends on $C_{\alpha}, C_{\beta}$, and $C^{\prime}$ defined above. In fact, this bound can be slightly improved since the squared error contributed by the wedgelet quantization can be bounded more tightly [31] to be less than or equal to $\delta$, rather that the loose bound of $O(\delta)$ we used above. Thus, the total squared error is bounded above by $K_{\alpha, \beta}^{\prime} m^{-\min (\alpha, \beta)}+\delta$.

\section{Proof of Computational Complexity Theorem}

Proof of Lemma 1 It is easy to check that the log multinomial likelihood function is concave in its parameters $(\rho=T \theta)$. To prove the lemma, we refer to Theorem 5.7 in [46], which states that if $T$ is a linear transformation from $\mathbb{R}^{N}$ to $\mathbb{R}^{M}$, then, for each convex function $g$ on $\mathbb{R}^{M}$, the function $g T$ defined by

$$
(g T)(\theta) \equiv g(T \theta)
$$

is convex in $\theta$ on $\mathbb{R}^{N}$. This and the concavity of multinomial log likelihood shows that

$$
L(x \mid \theta) \equiv \log \operatorname{Multinomial}(x \mid T \theta)
$$

is concave $\theta$.

Proof of Theorem 2 In order to calculate a Haar MPLE, one may obtain the sums of the counts in each dyadic square by performing a Haar wavelet analysis, which requires $O\left(N^{2}\right)$ calculations. Once the tree has been built with these parameters, the tree-pruning takes only $O\left(N^{2}\right)$ operations, yielding a total complexity of $O\left(N^{2}\right)$.

For wedgelets, note that there are $O\left(N^{2}\right)$ possible wedgelets to consider for an $N \times N$ pixel image. Direct calculation of the log likelihood for each wedgelet term requires $O\left(N^{2}\right)$ operations. The overall number of computations would then be $O\left(N^{4}\right)$. This number can be significantly reduced by carefully considering the impact of calculating the likelihood for each possible wedgelet 
in a sequential order. Consider two possible wedgelets, one dividing the image into regions $A_{1}$ and $B_{1}$, and the other dividing the image into regions $A_{2}$ and $B_{2}$, which are constructed so that the boundary separating $A_{1}$ and $B_{1}$ is different from the boundary separating $A_{2}$ and $B_{2}$ by only one pixel sidelength in one coordinate of one boundary endpoint. This is depicted in Figure 9. The two wedgelets are very similar; in fact, the number of pixels which common to both regions $A_{1}$ and $B_{2}$ or $A_{2}$ and $B_{1}$ may be bounded as follows: since the sidelength of the image is 1 and therefore the sidelength of each pixel is $1 / N$, the image area between the two boundaries is upper bounded by $1 /(2 N)$. Since the area of each pixel is $1 / N^{2}$, we have that the number of pixels which are in both regions $A_{1}$ and $B_{2}$ or $A_{2}$ and $B_{1}$ is of $O(N / 2)$. Because the likelihood function is additive, once the likelihood of the image being separated into regions $A_{1}$ and $B_{1}$ has been calculated, we need only $O(N / 2)$ operations to calculate the likelihood of the image being separated into regions $A_{2}$ and $B_{2}$.

The complete complexity calculation must consider the number of likelihood calculations at each level of the quad tree described above. At the coarsest scale, there is one block containing $N^{2}$ pixels and $N^{2}$ possible wedgelets, resulting in $O\left(N^{3}\right)$ operations for that level. On the second level, there are four blocks containing $N^{2} / 4$ pixels each at $N^{2} / 4$ possible wedgelets for each, resulting in $\frac{1}{2} O\left(N^{3}\right)$ operations for that level. This sequence continues, and is upper bounded by $O\left(N^{3}\right) \cdot \sum_{i=0}^{\infty}\left(\frac{1}{2}\right)^{i}$, or $2 O\left(N^{3}\right)$ for the entire image. Such a simplification is not possible when numerically searching for the maximum (multinomial) likelihood platelet fit, since all data in a given square are necessary to perform the search. Performing this search results in an $O\left(N^{4}\right)$ algorithm. An approximate platelet fit can be constructed using least-squares. Although this is suboptimal, our experimental results demonstrate it is a close approximation to the optimal platelet. Using the least squares approximation has the advantage of reducing the total number of operations to $O\left(N^{3}\right)$. The statistics of the data needed to calculate the least squares platelet fit are $\sum_{i, j} x_{i, j}, \sum_{i, j} i \cdot x_{i, j}, \sum_{i, j} j \cdot x_{i, j}$, where the sums are over the indices of pixels in the square or wedge under consideration (the individual data are not required). As in the wedgelet case, these statistics may be updated for each sequential platelet in $O(N)$ operations. As before, 
this coupled with the quad tree structure yields a total complexity of $O\left(N^{3}\right)$.

\section{REFERENCES}

[1] Z. H. Cho, J. P. Jones, and M. Singh, Foundations of Medical Imaging, Wiley-Interscience, New York, 1993.

[2] D. Synder and M. Miller, Random Point Processes in Time and Space, New York: Springer-Verlag, 1991.

[3] J. B.Pawley, Ed., Handbook of Biological Confocal Microscopy, Plenum Press, New York, 1995.

[4] A. P. Dempster, N. M. Laird, and D. B. Rubin, "Maximum likelihood from incomplete data via the EM algorithm," J. Royal Stat. Soc., vol. 39, pp. 1-38, 1977.

[5] Y. Vardi, L. A. Shepp, and L. Kaufman, "A statistical model for positron emission tomography," J. Amer. Statist. Assoc., vol. 80, pp. 8-37, 1985.

[6] G. M. P. van Kempen, H. T. M. van der Voort, J. G. J. Bauman, and K. C. Strasters, "Comparing maximum likelihood estimation and constrained tikhonov-miller restoration," IEEE Engineering in Medicain and Biology Magazine, vol. 15, pp. $76-83,1996$.

[7] W. Richardson, "Bayesian-based iterative method of image restoration," J. Opt. Soc. of Am., vol. 62, pp. $55-59,1972$.

[8] T. Hebert and R. Leahy, "A generalized EM algorithm for 3-d Bayesian reconstruction from Poisson data using Gibbs priors," IEEE Trans. Med. Imaging, vol. 8, no. 2, pp. 194-202, 1989.

[9] P. J. Green, "Bayesian reconstruction from emission tomography data using a modified EM algorithm," IEEE Trans. Med. Imaging, vol. 9, no. 1, pp. 84-93, 1990.

[10] J. A. Fessler and A. O. Hero, "Penalized maximum-likelihood image reconstruction using space-alternating generalized EM algorithms," IEEE Trans. Image Processing, vol. 4, no. 10, pp. 1417-1429, 1995.

[11] A. R. Depierro, "A modified expectation maximization algorithm for penalized likelihood estimation in emission tomography," IEEE Trans. Med. Imaging, pp. 132-137, 1995.

[12] J. Liu and P. Moulin, "Complexity-regularized image denoising," IEEE Transactions on Image Processing, vol. 10 , no. 6 , pp. $841-851,2001$.

[13] P. Moulin and J. Liu, "Statistical imaging and complexity regularization," IEEE Transactions on Information Theory, vol. 46, no. 5, pp. $1762-1777,2000$.

[14] N. Saito, "Simultaneous noise suppression and signal compression using a library of orthonormal bases and the minimum description length criterion," Wavelets in Geophysics, Foufoula-Georgiou and Kumar (eds.), Academic Press, 1994.

[15] H. Krim and I.C. Schick, "Minmax description length for signal denoising and optimal representation," IEEE Trans. on Information Theory, vol. 45, no. 3, April, 1999.

[16] G. McLachlan and T. Krishnan, The EM Algorithm and Extensions, Wiley, New York, 1997. 
[17] J. Llacer and E. Veklerov, "Feasible images and practical stopping rules for iterative algorithms in emission tomography," IEEE Trans. Med. Imaging, vol. 8, pp. 186-193, 1989.

[18] S. Mallat, A Wavelet Tour of Signal Processing, Academic Press, San Diego, CA, 1998.

[19] J. Starck, F. Murtagh, and A. Bijaoui, Image Processing and Data Analysis: The Multiscale Approach, Cambridge Univ. Press, 1998.

[20] A. Aldroubi and M. Unser, "Wavelets in medicine and biology," CRC Pr., Boca Raton FL, 1996.

[21] M. Bhatia, W. C. Karl, and A. S. Willsky, "A wavelet-based method for multiscale tomographic reconstruction," IEEE Trans. Med. Imaging, vol. 15, no. 1, pp. 92-101, 1996.

[22] E. D. Kolaczyk, "Wavelet shrinkage estimation of certain Poisson intensity signals using corrected thresholds," Statistica Sinica, vol. 9, pp. 119-135, 1999.

[23] N. Lee and B. J. Lucier, "Wavelet methods for inverting the radon transform with noisy data," IEEE Trans. Image Proc., vol. 10, pp. $79-94,2001$.

[24] J. Lin, A. F. Laine, and S. R. Bergmann, "Improving pet-based physiological quantification through methods of wavelet denoising," IEEE Trans. Bio. Eng., vol. 48, pp. $202-212,2001$.

[25] N. Kingsbury, "Image processing with complex wavelets," Phil. Trans. Royal Society London A, vol. 357, pp. 2543-2560, 1999.

[26] E. P. Simoncelli and W. T. Freeman, "The steerable pyramid: A flexible architecture for multi-scale derivative computation," in International Conference on Image Processing, 23-26 Oct. 1995, Washington, DC, USA, 1995, vol. 3, pp. 444-447.

[27] E. Candès and D. Donoho, "Curvelets: A surprisingly effective nonadaptive representation for objects with edges," To appear in Curves and Surfaces, L. L. Schumaker et al. (eds), Vanderbilt University Press, Nashville, TN.

[28] E. Kolaczyk and R. Nowak, "A multiresolution analysis for likelihoods: Theory and methods," submitted to Annals of Stat. Available at http://www.ece.rice.edu/ nowak/publications.html.

[29] K. Timmermann and R. Nowak, "Multiscale modeling and estimation of Poisson processes with application to photon-limited imaging," IEEE Transactions on Information Theory, vol. 45, no. 3, pp. 846-862, April, 1999.

[30] E. Kolaczyk, "Bayesian multi-scale models for Poisson processes," J. Amer. Statist. Assoc., vol. 94, pp. 920-933, 1999.

[31] D. Donoho, "Wedgelets: Nearly minimax estimation of edges," Ann. Statist., vol. 27, pp. 859 - $897,1999$.

[32] E. Kolaczyk and R. Nowak, "Risk analysis for multiscale penalized maximum likelihood estimators," submitted to Annals of Stat. Available at http://www.ece.rice.edu/ nowak/publications.html.

[33] D. Donoho, "Cart and best-ortho-basis selection: A connection," Annals of Stat., vol. 25, pp. 1870-1911, 
1997.

[34] H. Triebel, Interpolation Theory, Function Spaces, Differential Operators, North-Holland, Amsterdam, 1978.

[35] D. Donoho, "Sparse components of images and optimal atomic decompositions," Constr. Approx., vol. 17, pp. 353-382, 2001.

[36] R. R. Coifman, Y. Meyer, S. Quake, and M. V. Wickerhauser, "Wavelet analysis and signal processing," in Wavelets and Their Applications, J. S. Byrnes, J. L. Byrnes, K. A. Hargreaves, and K. Berry, Eds. Kluwer Academic, Boston, 1994.

[37] M. Unser and M. Eden, "Maximum likelihood estimation of linear signal parameters for poisson processes," IEEE Transactions on Acoustics, Speech, and Signal Processing, vol. 36, no. 6, pp. 942-5, 1988.

[38] R. Nowak and R. Baraniuk, "Wavelet-domain filtering for photon imaging systems," IEEE Transactions on Image Processing, vol. 8, no. 5, 1999.

[39] R. Coifman and D. Donoho, "Translation invariant de-noising," in Lecture Notes in Statistics: Wavelets and Statistics, vol. New York: Springer-Verlag, pp. 125-150, 1995.

[40] M. Lang, H. Guo, J. E. Odegard, C. S. Burrus, and R. O. Wells, "Noise reduction using an undecimated discrete wavelet transform," IEEE Signal Processing Letters, vol. 3, no. 1, pp. 10-12, 1996.

[41] L. A. Shepp and Y. Vardi, "Maximum likelihood reconstruction for emission tomography," IEEE Trans. Med. Imaging, vol. 1, pp. 113-122, 1982.

[42] R. Nowak and E. Kolaczyk, "A multiscale statistical framework for Poisson inverse problems," IEEE Trans. Info. Theory, vol. 46, pp. 1811-1825, 2000.

[43] K. E. Sorra and K. M. Harris, "Overview on the structure, composition, function, development, and plaasticity of hippocampal dendritic spines," Hippocampus, vol. 10, pp. 501-511, 2000.

[44] J. A. Fessler, "Aspire 3.0 user's guide: A sparse iterative reconstruction library," Communication \& Signal Processing Laboratory Technical Report No. 293, Department of Electrical and Computer Engineering, University of Michigan, Ann Arbor, 1998.

[45] D. L. Donoho, "Nonlinear solution of linear inverse problems by wavelet-vaguelette decomposition," App. and Comp. Harmonic Analysis, vol. 2, pp. 101-126, 1995.

[46] T. R. Rockafellar, Convex Analysis, Princeton University Press, Princeton, New Jersey, 1972. 
(a)

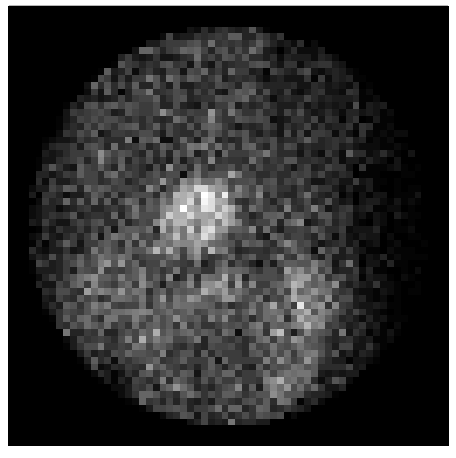

(b)

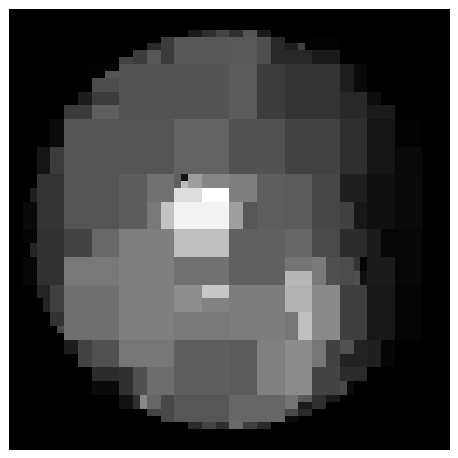

(d)

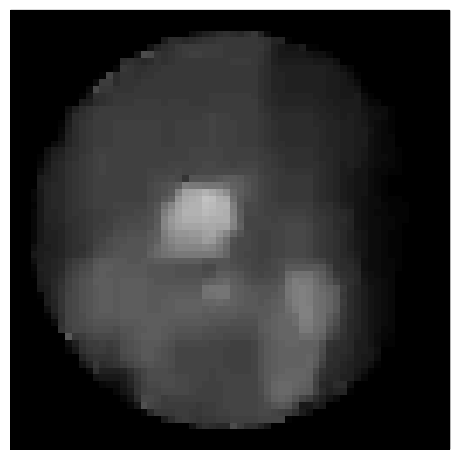

(e)

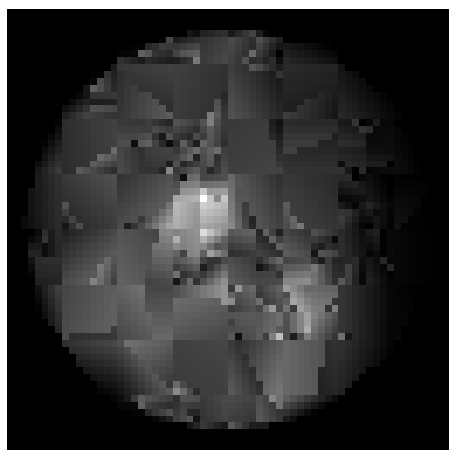

(c)

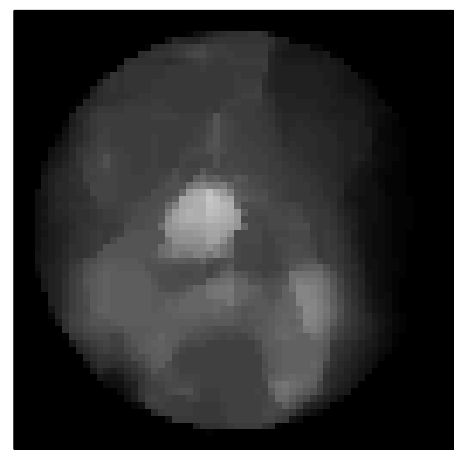

(g)

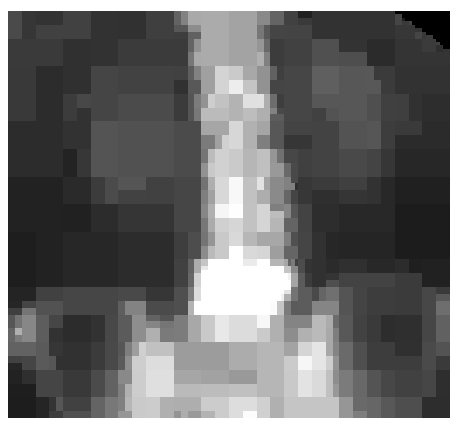

(i)

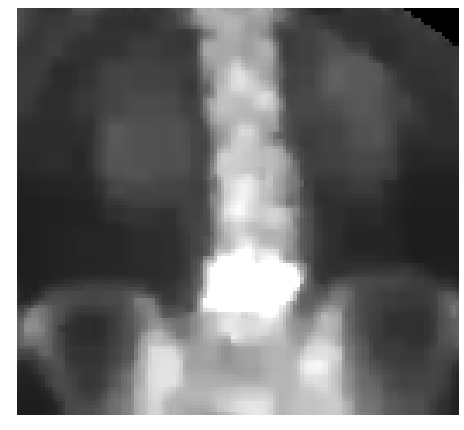

(h)

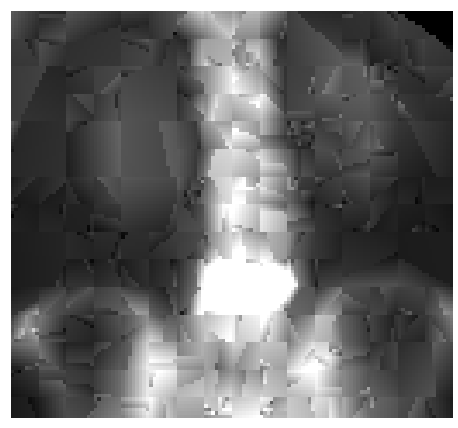

(j)

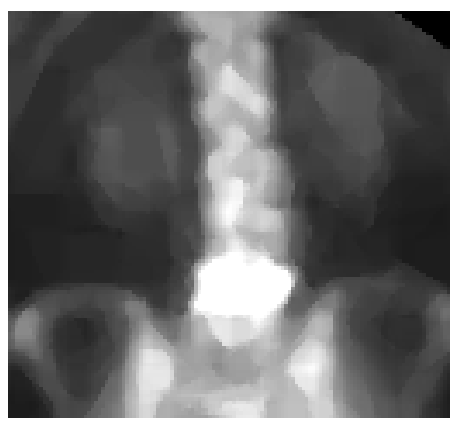

Fig. 5. Denoising in nuclear medicine. (a) "Raw" nuclear medicine cardiac image $(64 \times 64$ pixels). (b) Haar-based MPLE, $\beta=1 / 5$. (c) Platelet-based MPLE, $\beta=1 / 5$. (d) Haar-based MPLE, $\beta=1 / 5$, averaged over 25 shifts. (e) Platelet-based MPLE, $\beta=1 / 5$, averaged over 25 shifts. (f) "Raw" nuclear medicine spine image $(256 \times 256$ pixels $)$. (g) Haar-based MPLE, $\beta=1 / 5$. (h) Platelet-based MPLE, $\beta=1 / 5$. (i) Haar-based MPLE, $\beta=1 / 5$, averaged over 25 shifts. (j) Platelet-based MPLE, $\beta=1 / 5$, averaged over 25 shifts. 
(a)

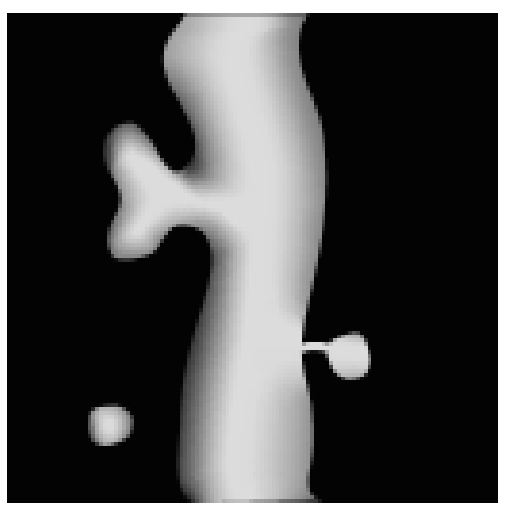

(d)

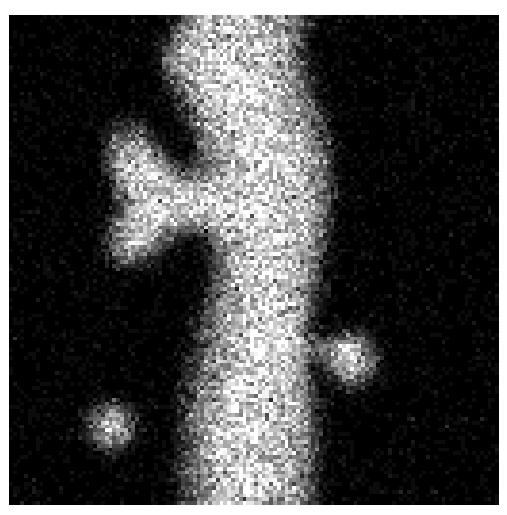

(g)

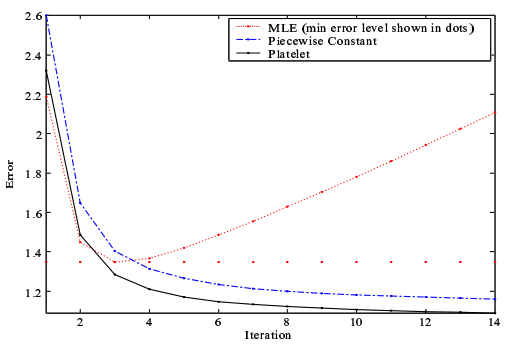

(b)

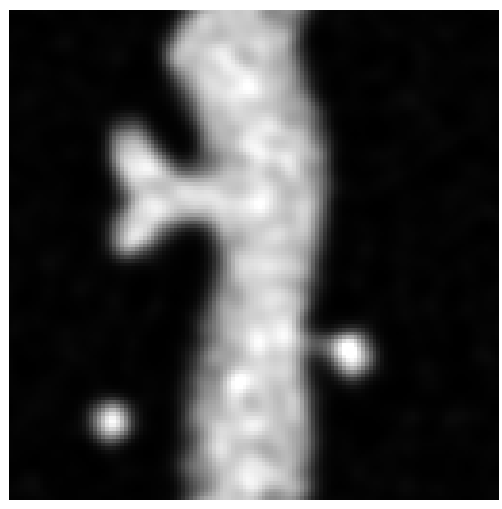

(e)

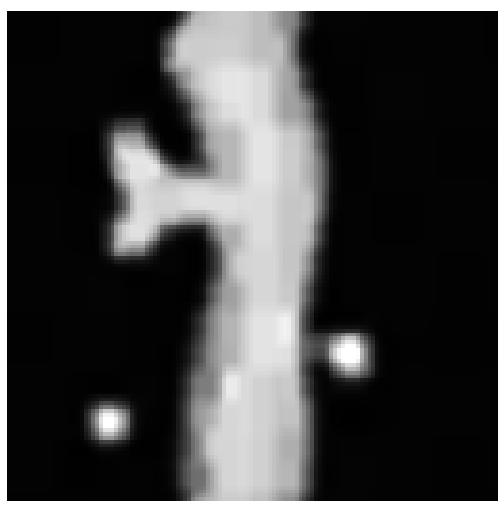

(h)

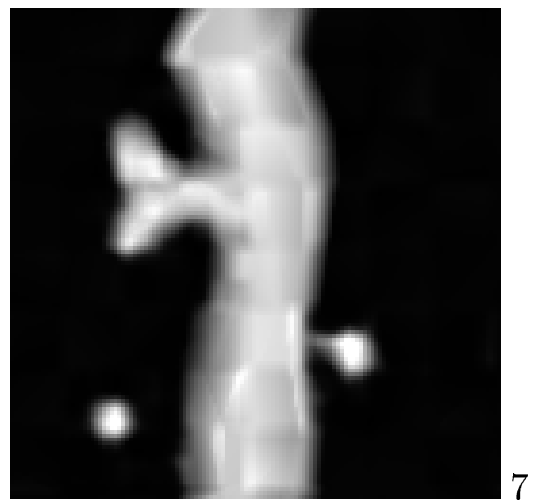

(c)

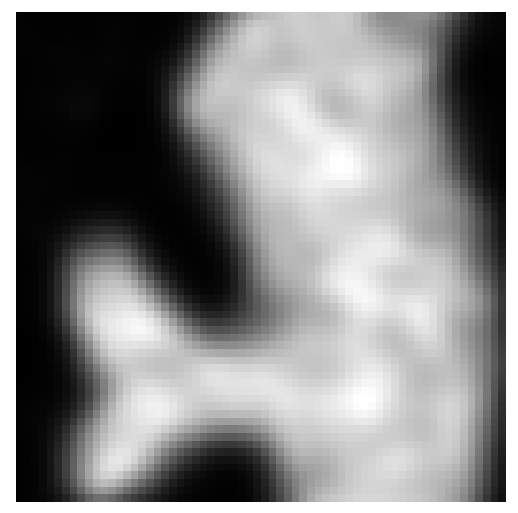

(f)

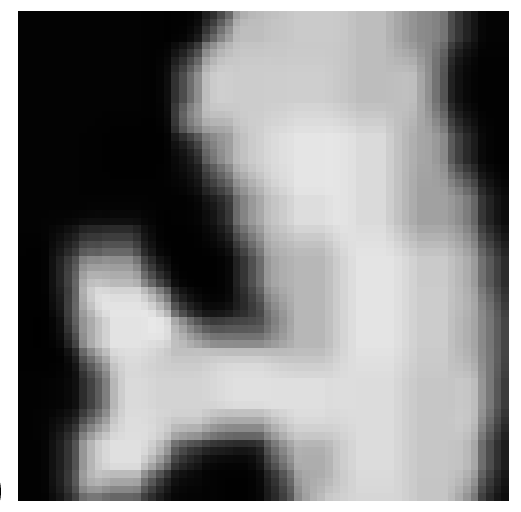

(i)

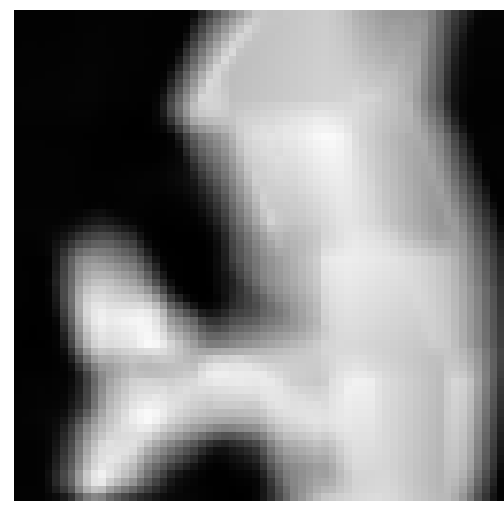

Fig. 6. Confocal Microscopy Simulation. (a) Phantom $(128 \times 128$ pixels), (b) Best EM-MLE restoration, (c) Best EM-MLE (Zoomed), (d) Blurred and Noisy Phantom, (e) Hereditary Haar MPLE (averaged over $3 \times 3$ shifts), (f) Hereditary Haar MPLE (Zoomed), (g) Error Decay by Iteration, (h) Platelet MPLE (averaged over $3 \times 3$ shifts), (i) Platelet MPLE (Zoomed). In all cases, convergence was declared when $\left\|\boldsymbol{\lambda}^{(i+1)}-\boldsymbol{\lambda}^{(i)}\right\|_{2} /\left\|\boldsymbol{\lambda}^{(i)}\right\|_{2}<10^{-5}$ (14 iterations in this case). 
(a)

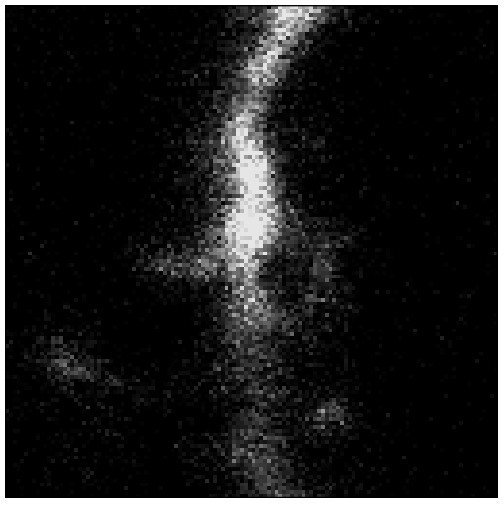

(c)

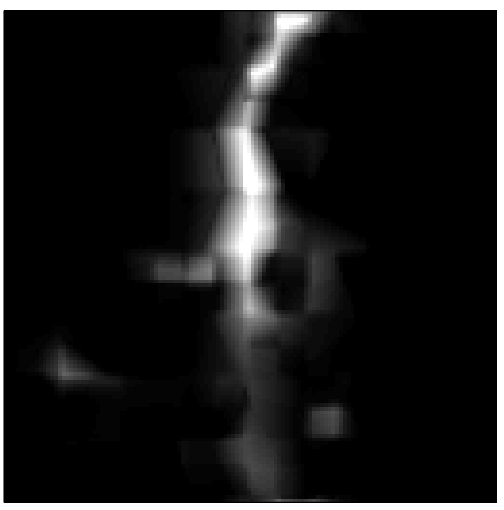

(b)
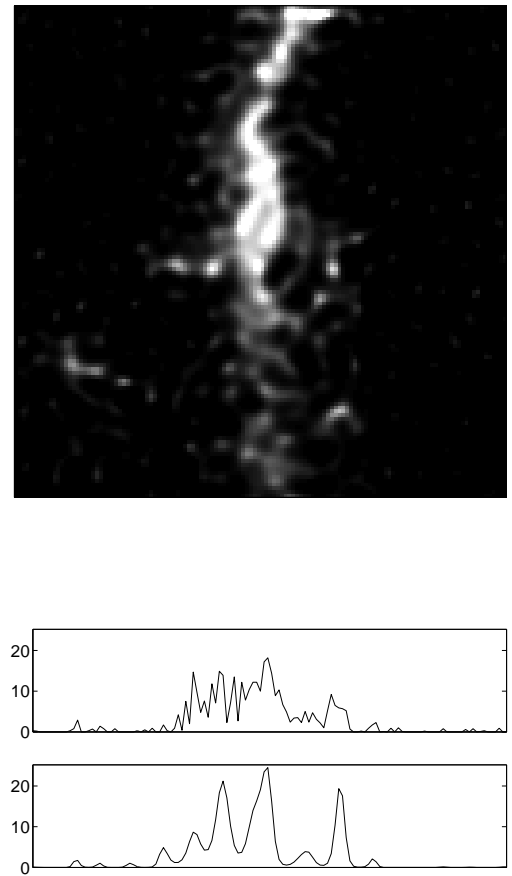

(d)

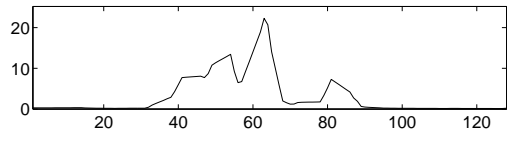

Fig. 7. Confocal Microscopy Application (a) Raw Data, (b) Best EM-MLE restoration, (c) Platelet-based MPLE (averaged over $3 \times 3$ shifts). In all cases, convergence was declared when $\left\|\boldsymbol{\lambda}^{(i+1)}-\boldsymbol{\lambda}^{(i)}\right\|_{2} /\left\|\boldsymbol{\lambda}^{(i)}\right\|_{2}<$ $10^{-5}$ (11 iterations in this case). (d) Profiles (row 70) of all three images (raw data, MLE, MPLE from top to bottom). Note that the MLE oscillates wildly, whereas the MPLE is much more well behaved. 
(a)

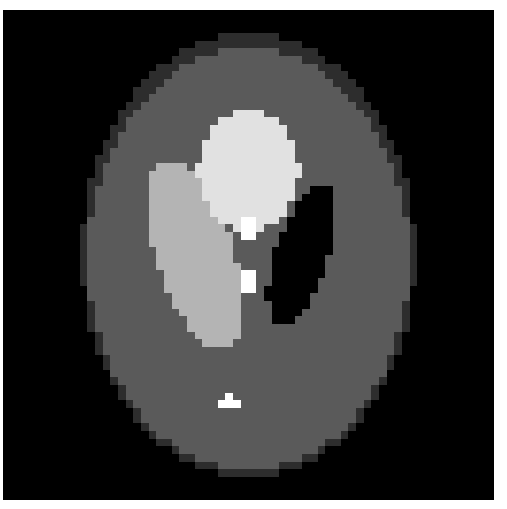

(c)

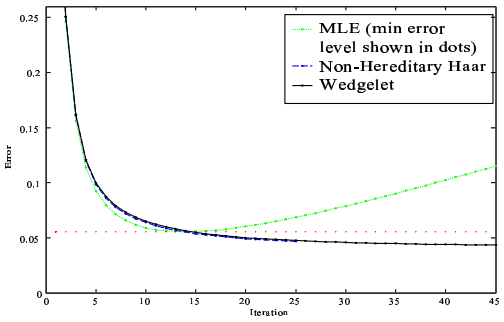

(b)

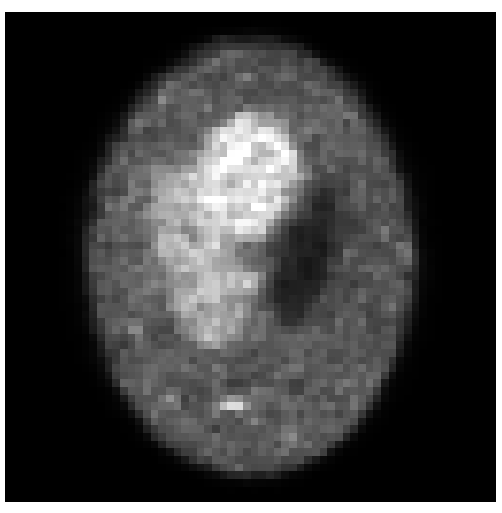

(d)

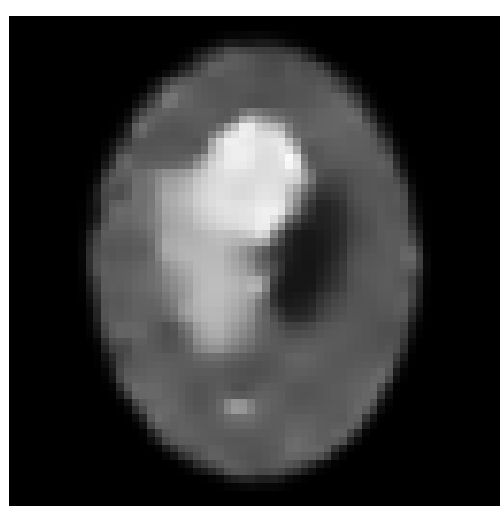

(e)

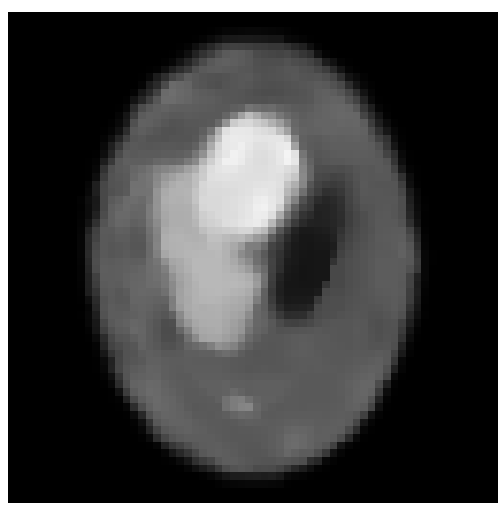

Fig. 8. SPECT Simulation. (a) Shepp-Logan phantom $(64 \times 64)$, (b) Best EM-MLE reconstruction (stopped at 14 iterations which gave the minimum squared error reconstruction), (c) Error vs. iteration for all three methods. (d) Non-Hereditary Haar-based MPLE (averaged over $5 \times 5$ shifts), (e) Wedgelet-based MPLE (averaged over $5 \times 5$ shifts). In all cases, convergence was declared when $\left\|\boldsymbol{\lambda}^{(i+1)}-\boldsymbol{\lambda}^{(i)}\right\|_{2} /\left\|\boldsymbol{\lambda}^{(i)}\right\|_{2}<$ $10^{-5}$ (roughly 25 iterations in these cases.) 

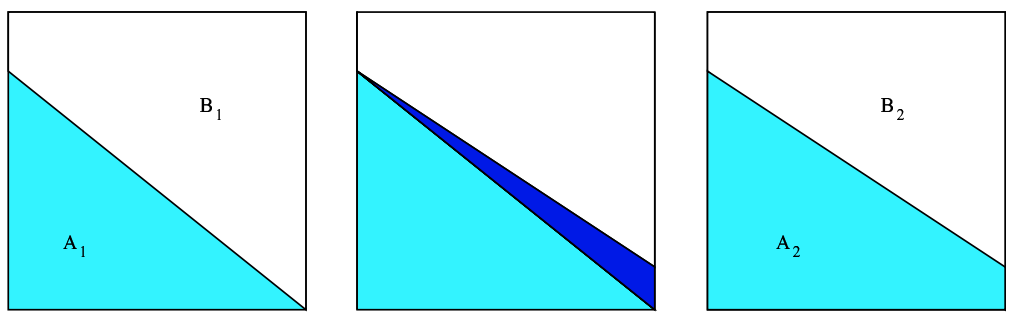

Fig. 9. Sequential Calculation of Wedgelet Likelihoods 